\title{
Quantum Graphs and Random-Matrix Theory
}

\author{
Z. Pluhař $\check{\check{r}}^{a}$ and H. A. Weidenmüller ${ }^{b}$ \\ ${ }^{a}$ Faculty of Mathematics and Physics, Charles University, \\ 18000 Praha 8, Czech Republic \\ ${ }^{b}$ Max-Planck-Institut für Kernphysik, \\ 69029 Heidelberg, Germany
}

October 4, 2018

\begin{abstract}
For simple connected graphs with incommensurate bond lengths and with unitary symmetry we prove the Bohigas-Giannoni-Schmit conjecture in its most general form. Using supersymmetry and taking the limit of infinite graph size, we show that the generating function for every $(P, Q)$ correlation function for both closed and open graphs coincides with the corresponding expression of random-matrix theory. We use that the classical Perron-Frobenius operator is bistochastic and possesses a single eigenvalue +1 . In the quantum case that implies the existence of a zero (or massless) mode of the effective action. That mode causes universal fluctuation properties. Avoiding the saddle-point approximation we show that for graphs that are classically mixing (i.e., for which the spectrum of the classical Perron-Frobenius operator possesses a finite gap) and that do not carry a special class of bound states, the zero mode dominates in the limit of infinite graph size.
\end{abstract}

\section{Introduction}

The distribution of eigenvalues and eigenfuctions of a classically chaotic Hamiltonian quantum system forms one of the central topics of quantum chaos. The celebrated conjecture by Bohigas, Giannoni and Schmit (BGS) [1] (see also Refs. 2, 3, 4]) states that the spectral fluctuation properties of a Hamiltonian quantum system that is classically chaotic (mixing) coincide with those of the random-matrix ensemble in the same symmetry class. Here the words "spectral fluctuation properties" comprise the totality of spectral fluctuation measures. The symmetry class (orthogonal, unitary, or symplectic) is determined [5] by the properties of the system under time reversal and under rotation.

Since 1984 the conjecture has found ample numerical support, see Ref. 6] and references therein. However, it took about twenty years for the first analytical evidence to appear in its favor. In Refs. [7, 8, 9, 10, 11] the semiclassical 
approximation in the form of Gutzwiller's periodic-orbit theory [12] was continuously refined to eventually yield a convincing demonstration of the equality of the level-level correlator ("two-point function") of a chaotic (hyperbolic) system and that of random-matrix theory (RMT), both for unitary and orthogonal symmetry. The equality holds within an energy interval defined by the period of the shortest periodic orbit. A parallel effort was devoted to quantum graphs [13. As explained below, these systems, while not strictly Hamiltonian, are semiclassical from the outset. In Refs. [14, 15] the two-point function for closed quantum graphs was shown to coincide with that of RMT, both for unitary and orthogonal symmetry. Open time-reversal-invariant graphs were considered in Refs. [16, 17]. It was shown that the correlation function of a pair of elements of the scattering matrix ( $S$ matrix) is equal to that given by the RMT approach of Ref. [18. As a by-product, the complete distribution function (given by its moments) of the $S$ matrix in the Ericson regime (strongly overlapping resonances) was obtained. In the absence of a corresponding result for RMT it was conjectured that this result is universal, too.

In this paper we present a proof of the BGS conjecture for quantum graphs in its most general form. We show that each level correlator for closed graphs and each S-matrix correlator for open graphs coincides with the corresponding expression for RMT red in the limit of infinite graph size. Not being able to work out these correlators in general, we demonstrate the equality by showing that their generating functions are pairwise identical. We define the interval of wave numbers wherein the equality holds. Since Ref. [19] contains a brief account of our results for the orthogonal case, we focus attention in the present paper on the unitary case. As a by-product we prove the above-mentioned conjecture concerning Ericson fluctuations formulated in Refs. [16, 17.

\section{Quantum Graphs}

A closed graph [13, 20] is a set of $V$ vertices labeled $\alpha=1, \ldots, V$ that are connected by $B$ bonds labeled $b=1, \ldots, B$. For uniqueness we label the bonds also by the indices $(\alpha \beta)$ which denote the pair of vertices to which the bond is attached. We consider simple connected graphs. In a simple graph, every pair of non-identical vertices is connected by at most a single bond, and every bond connects a pair of non-identical vertices. In a connected graph, starting from any vertex $\alpha$ it is possible to reach any other vertex $\beta$ through a chain of bonds all of which belong to the graph. The bond lengths $L_{b}$ are assumed to be incommensurate (there exists no linear combination with integer coefficients $i_{b}$ such that $\left.\sum_{b} i_{b} L_{b}=0\right)$. Let $B_{\alpha}$ denote the number of bonds issuing from vertex $\alpha$. Then the total number of bonds is $B=(1 / 2) \sum_{\alpha=1}^{V} B_{\alpha}$. We are interested in generic features and, therefore, consider the limit $B \rightarrow \infty$ of infinite graph size. We assume that in that limit, the bond lengths remain bounded so that $L_{\min } \leq L_{b} \leq L_{\max }$ with finite $L_{\min }, L_{\max }$ for all $b$. On each bond the Schrödinger wave has the form $s_{b 1} \exp \left\{i k x_{b}\right\}+s_{b 2} \exp \left\{-i k x_{b}\right\}$ where $x_{b}$ denotes the distance to one of the two vertices attached to the bond, and where the wave number $k$ 
has the same value on all bonds. The set of coefficients $\left\{s_{b 1}, s_{b 2}\right\}$ is determined by Hermitean boundary conditions imposed at each vertex. As a result the vector $\mathcal{I}^{(\alpha)}$ of incoming waves on all $B_{\alpha}$ bonds attached to vertex $\alpha$ and the vector $\mathcal{O}^{(\alpha)}$ of outgoing waves on the same bonds are related by $\mathcal{O}^{(\alpha)}=\sigma^{(\alpha)} \mathcal{I}^{(\alpha)}$. The matrices $\sigma^{(\alpha)}$ [20] have dimension $B_{\alpha}$, are unitary (flux conservation) and, for time-reversal invariant graphs, are symmetric.

An open graph is obtained from a closed graph as defined in the previous paragraph by attaching to each one of $\Lambda$ vertices an additional single bond that extends to infinity. Without loss of generality these vertices are labeled $\alpha=1, \ldots, \Lambda$. The attached additional bonds carry the same labels $\alpha=1, \ldots, \Lambda$ and are referred to as channels. We keep the number of channels $\Lambda$ fixed when we let $B \rightarrow \infty$. Hermitean boundary conditions imposed on all vertices yield the relations $\mathcal{O}^{(\alpha)}=\Gamma^{(\alpha)} \mathcal{I}^{(\alpha)}$. Here $\mathcal{I}^{(\alpha)}$ and $\mathcal{O}^{(\alpha)}$ are the vectors of incoming and outgoing amplitudes on all the bonds (channels) attached to vertex $\alpha$. For $\alpha \leq \Lambda(\alpha>\Lambda)$ these vectors and the matrices $\Gamma^{(\alpha)}$ have dimension $\left(B_{\alpha}+1\right)\left(B_{\alpha}\right.$, respectively). The matrices $\Gamma^{(\alpha)}$ are unitary and, for time-reversal invariant graphs, are symmetric. We write the matrices $\Gamma^{(\alpha)}$ in the form

$$
\begin{aligned}
& \Gamma^{(\alpha)}=\left(\begin{array}{ll}
\rho^{(\alpha)} & \tau_{\beta}^{(\alpha)} \\
\tilde{\tau}_{\gamma}^{(\alpha)} & \sigma_{\gamma \beta}^{(\alpha)}
\end{array}\right) \text { for } \alpha \leq \Lambda, \\
& \Gamma^{(\alpha)}=\left(\sigma_{\gamma \beta}^{(\alpha)}\right) \text { for } \alpha>\Lambda .
\end{aligned}
$$

The coefficient $\rho^{(\alpha)}$ defines the amplitude for backscattering from channel $\alpha$ into channel $\alpha$. The coefficient $\tau_{\beta}^{(\alpha)}\left(\tilde{\tau}_{\gamma}^{(\alpha)}\right)$ defines the amplitude for scattering from bond $(\alpha \beta)$ to channel $\alpha$ (from channel $\alpha$ to bond $(\alpha \gamma)$, respectively). The matrices $\sigma^{(\alpha)}$ have dimension $B_{\alpha}$, are subunitary (unitary) for $\alpha \leq \Lambda$ (for $\alpha>\Lambda$, respectively) and, for time-reversal invariant graphs, are symmetric.

Every set of Hermitean boundary conditions defines a set of unitary matrices $\left\{\sigma^{(\alpha)}\right\}$ or $\left\{\Gamma^{(\alpha)}\right\}$, as the case may be. The converse is not neccessarily true. In constructing the theory we do not specify the boundary conditions but work with an arbitrary set of unitary matrices $\left\{\sigma^{(\alpha)}\right\}$ or $\left\{\Gamma^{(\alpha)}\right\}$. That is legitimate: All conclusions drawn for that set hold also for graphs defined by Hermitean boundary conditions.

\subsection{Wave Propagation through a Graph}

Expressions for the spectral determinant and for the scattering matrix of graphs have been derived, for instance, in Refs. 13, 20. For brevity we confine ourselves to a heuristic argument that highlights the essential points without any claim to rigor. In a perturbative approach to multiple vertex scattering, the amplitude $\tilde{\mathcal{W}}_{\beta \alpha}^{-1}$ for wave propagation from vertex $\alpha$ to vertex $\beta$ has the form

$$
\begin{aligned}
& \tilde{\mathcal{W}}_{\beta \alpha}^{-1}=\exp \left\{i k L_{\beta \alpha}\right\} \\
& +\sum_{\gamma} \exp \left\{i k L_{\beta \gamma}\right\} \sigma_{\beta \alpha}^{(\gamma)} \exp \left\{i k L_{\gamma \alpha}\right\}
\end{aligned}
$$




$$
\begin{aligned}
& +\sum_{\gamma \delta} \exp \left\{i k L_{\beta \gamma}\right\} \sigma_{\beta \delta}^{(\gamma)} \exp \left\{i k L_{\gamma \delta}\right\} \sigma_{\gamma \alpha}^{(\delta)} \exp \left\{i k L_{\delta \alpha}\right\} \\
& +\ldots
\end{aligned}
$$

To sum that series we introduce matrix notation. The block-diagonal vertex scattering matrix $\Sigma^{(V)}$ connects incoming and outgoing amplitudes on all vertices. It carries the $V$ matrices $\sigma^{(\alpha)}$ with $\alpha=1,2, \ldots, V$ in its diagonal blocks. By definition, $\Sigma^{(V)}$ is unitary for closed and subunitary for open graphs and has dimension $2 B$, twice the number $B$ of bonds. Therefore, a doubling of bond indices is indicated. To that end we introduce "directed" bonds. We arrange the $B$ bonds $(\alpha \beta)$ in lexicographical order so that $\alpha<\beta$. The resulting series is mapped onto the sequence $b=1, \ldots, B$ of integers. The directed bonds in the series are labeled $(b+)$. To every such directed bond $(\alpha \beta)$ with $\alpha<\beta$ we associate the bond $(\beta \alpha)$ with opposite direction and denote it by $(b-)$. With $d= \pm$ the totality of $2 B$ directed bonds is labeled $(b d)$. In directed-bond representation the matrix $\sigma^{(\alpha)}$ with elements $\sigma_{\beta \gamma}^{(\alpha)}$ is written as $\sigma_{\alpha \beta, \alpha \gamma}=\sigma_{b d, b^{\prime} d^{\prime}}$, with the bond labels $b\left(b^{\prime}\right)$ determined by $(\alpha \beta)$ (by $(\alpha \gamma)$, respectively), with $d$ positive (negative) for $\alpha<\beta$ (for $\alpha>\beta$, respectively), and correspondingly for $d^{\prime}$. When written in directed-bond representation, the vertex scattering matrix $\Sigma^{(V)}$ becomes the "bond scattering matrix" $\Sigma^{(B)}$ with elements $\Sigma_{b d, b^{\prime} d^{\prime}}^{(B)}$. The map $\Sigma^{(V)} \rightarrow \Sigma^{(B)}$ involves an identical rearrangement of rows and columns. Therefore, $\Sigma^{(B)}$ is also unitary (subunitary) for closed (open) graphs, respectively. For time-reversal invariant graphs, $\Sigma^{(B)}$ is symmetric. The diagonal matrix $\exp \{i k \mathcal{L}\}$ with $\mathcal{L}_{b d, b^{\prime} d^{\prime}}=L_{b} \delta_{b b^{\prime}} \delta_{d d^{\prime}}$ describes amplitude propagation on the directed bonds. Eq. (2) can be summed to give

$$
\tilde{\mathcal{W}}=\exp \{-i k \mathcal{L}\}-\sigma_{1}^{d} \Sigma^{(B)},
$$

with $\sigma_{1}^{d}$ the first Pauli spin matrix in directed bond space. Eq. (3) is verified by expanding $\tilde{\mathcal{W}}^{-1}$ in powers of $\Sigma^{(B)}$. The factor $\sigma_{1}^{d}$ is required because of the definition of $\sigma^{(\alpha)}$ in directed-bond representation given above. The connection with the definitions used in Refs. [16, 17, 19] is established by defining

$$
\mathcal{W}=\sigma_{1}^{d} \tilde{\mathcal{W}}=\sigma_{1}^{d} \exp \{-i k \mathcal{L}\}-\Sigma^{(B)} .
$$

We note that $\tilde{\mathcal{W}}$ and $\mathcal{W}$ carry the complete information on wave propagation through the graph. Therefore, both the spectral determinant and the scattering matrix can be written in terms of these matrices. The spectral determinant $\xi(k)$ is 14,15

$$
\begin{aligned}
\xi(k) & =\operatorname{det}\{\exp \{i k \mathcal{L}\} \tilde{\mathcal{W}}\} \\
& =\operatorname{det}\left\{1-\exp \{i k \mathcal{L}\} \sigma_{1}^{d} \Sigma^{(B)}\right\}
\end{aligned}
$$

Zeros of $\xi(k)$ at $k=k_{n}$ with $n=1,2, \ldots$ define the bound states of the graph. The level density $d(k)$ of the graph is given by [15]

$$
d(k)=\sum_{n} \delta\left(k-k_{n}\right)=\left\langle d_{R}\right\rangle+d^{\mathrm{f}}(k)
$$


where

$$
\left\langle d_{R}\right\rangle=\frac{1}{\Delta}=\frac{1}{\pi} \sum_{b} L_{b}
$$

is the average level density and where $\Delta$ denotes the mean level spacing. The fluctuating part $d^{\mathrm{f}}(k)$ of the level density is given by [15]

$$
d^{\mathrm{f}}(k)=-\frac{1}{2 i \pi} \frac{\mathrm{d}}{\mathrm{d} k}\left(\ln \xi\left(k^{+}\right)-\ln \xi\left(k^{-}\right)\right) .
$$

Here $k^{ \pm}=k \pm i \epsilon$ with $\epsilon>0$ and infinitesimal. Scattering on the graph is described by the scattering matrix $S(k)$, a function of the wave number $k$. The amplitude $S_{\beta \alpha}(k)$ for scattering from channel $\alpha$ into channel $\beta$ is given by [21]

$$
S_{\beta \alpha}(k)=\rho^{(\alpha)} \delta_{\alpha \beta}+\sum_{\gamma \delta} \tau_{\gamma}^{(\beta)} \tilde{\mathcal{W}}_{\gamma \delta}^{-1} \tilde{\tau}_{\delta}^{(\alpha)}
$$

In directed-bond representation, the matrices $\tau_{\gamma}^{(\beta)}$ are written as $\tau_{\beta, \beta \gamma}=\tau_{\beta, b d}$ with $(b d)$ determined by $(\beta \gamma)$ in terms of the rules stated above. The totality of these matrices forms the rectangular matrix $\mathcal{T}$ with $\Lambda$ rows and $2 B$ columns. The matrices $\tilde{\tau}_{\delta}^{(\alpha)}$ are similarly written as $\tilde{\tau}_{\alpha \delta, \alpha}=\tilde{\tau}_{b d, \alpha}$. The totality of these matrices forms a rectangular matrix $\tilde{\mathcal{T}}$ with $2 B$ rows and $\Lambda$ columns. With these definitions the $S$ matrix in Eq. (9) takes the form

$$
S(k)=\rho+\mathcal{T} \mathcal{W}^{-1} \tilde{\mathcal{T}} .
$$

Here $\rho$ is diagonal in channel space with elements $\rho^{(\alpha)}$. The average $S$ matrix $\langle S\rangle$ is given by 13 .

$$
\langle S\rangle=\rho .
$$

The fluctuating part $S^{\mathrm{fl}}$ of $S$ is accordingly given by

$$
S^{\mathrm{fl}}(k)=\mathcal{T} \mathcal{W}^{-1} \tilde{\mathcal{T}} .
$$

For $\alpha \leq \Lambda$ the unitarity of $\Gamma^{(\alpha)}$ in Eqs. (1) implies

$$
\left(\sigma^{(\alpha) \dagger} \sigma^{(\alpha)}\right)_{\beta \gamma}=\delta_{\beta \gamma}-\tau_{\beta}^{(\alpha) *} \tau_{\gamma}^{(\alpha)} .
$$

The transmission coefficient

$$
T^{(\alpha)}=\sum_{\beta}\left|\tau_{\beta}^{(\alpha)}\right|^{2}=1-\left|\rho^{(\alpha)}\right|^{2}
$$

measures the unitarity deficit of the average $S$ matrix in Eq. (11).

\subsection{Chaotic Graphs}

We consider closed graphs that are chaotic in the classical limit [22, 23, 20]. In that limit, amplitudes are replaced by probabilities, and interest centers on 
the $(2 B)$-dimensional vector $r$ of occupation propabilities $r_{b d} \geq 0$ for the $2 B$ directed bonds $(b d)$. The discrete time evolution of $r$ is given by the map $r \rightarrow \mathcal{F} r$. Here $\mathcal{F}$ is the Perron-Frobenius operator 20, a non-symmetric matrix in directed-bond space with elements $\mathcal{F}_{b d, b^{\prime} d^{\prime}}=\left|\left(\sigma_{1}^{d} \Sigma^{(B)}\right)_{b d, b^{\prime} d^{\prime}}\right|^{2}$. The classical motion is chaotic (mixing) if for large times the vector $r$ approaches the uniform distribution on the set of $2 B$ directed bonds exponentially fast. That is the case if the spectrum of $\mathcal{F}$ obeys certain requirements.

By definition, all elements of $\mathcal{F}$ are positive or zero. Moreover, $\mathcal{F}$ is bistochastic, i.e., $\sum_{b^{\prime} d^{\prime}} \mathcal{F}_{b d, b^{\prime} d^{\prime}}=1=\sum_{b d} \mathcal{F}_{b d, b^{\prime} d^{\prime}}$. This follows from the unitarity of $\Sigma^{(B)}$ and from the form of $\sigma_{1}^{d}$. For connected graphs, the map $r \rightarrow \mathcal{F} r$ does not possess an invariant subspace, i.e., the matrix $\mathcal{F}$ is irreducible. For matrices with these properties the Perron-Frobenius theorem states that there exists a non-degenerate maximal eigenvalue $\lambda_{1}=1$ with associated normalized right (left) eigenvectors

$$
u_{1}=(1 / \sqrt{2 B})(1,1, \ldots, 1)^{T}, w_{1}=(1 / \sqrt{2 B})(1,1, \ldots, 1)
$$

All other eigenvalues $\lambda_{j}$ with $2 \leq j \leq 2 B$ obey $\left|\lambda_{j}\right| \leq 1$. The associated right (left) eigenvectors $u_{j}\left(w_{j}\right.$, respectively) obey $\left\langle w_{j} \mid u_{j^{\prime}}\right\rangle=\delta_{j j^{\prime}}$ for $j, j^{\prime}=1, \ldots, 2 B$. In general some of the eigenvalues $\lambda_{j}$ with $j \geq 2$ may lie on the unit circle in the complex plane. However, for the graph to be mixing [20, $\lambda_{1}$ must be the only eigenvalue on that circle. All other eigenvalues must lie within or on the surface of a disc within the unit circle. For an $m$-fold repeated map $r \rightarrow \mathcal{F}^{m} r=\sum_{j=1}^{2 B}\left(\lambda_{j}\right)^{m} u_{j}\left\langle w_{j} \mid r\right\rangle$ we then have $r \rightarrow u_{1}\left\langle w_{1} \mid r\right\rangle$ for $m \rightarrow \infty$, and the uniform distribution is attained exponentially fast. For the graph to remain mixing in the limit $B \rightarrow \infty$ we require that the minimum distance between the disc of eigenvalues $\lambda_{j}$ with $j \geq 2$ and the unit circle remains finite, $\left|\lambda_{j}\right| \leq 1-a$ with $a>0$ for $B \rightarrow \infty$.

For open graphs, the leading eigenvalue differs from unity, and the leading eigenvector differs from $u_{1}$ (from $w_{1}$, respectively). That difference is taken into account explicitly in our calculation, see Section 3.3. We postulate also for open graphs that the remaining eigenvalues $\lambda_{j}$ with $j \geq 2$ obey $\left|\lambda_{j}\right| \leq 1-a$ with $a>0$ and that this relation remains valid for $B \rightarrow \infty$.

\subsection{Correlation Functions}

The fluctuation properties of graphs are completely determined by the set of all correlation functions. These functions are defined as averages over $k$ (indicated by angular brackets) and, for closed graphs, are given by $\left\langle\prod_{i=1}^{N} d^{\mathrm{f}}\left(k_{i}\right)\right\rangle$ with $N=2,3, \ldots$, taken at arguments $k_{1}, k_{2}, \ldots, k_{N}$. For open graphs, they are correspondingly given by averages over products of $N$ fluctuating $S$-matrix elements or their complex conjugates taken at arguments $k_{1}, k_{2}, \ldots, k_{N}$. We use the well-known fact (see also below) that for all $k_{1}, k_{2}, \ldots, k_{N}$ we have

$$
\left\langle\left.\prod_{i=1}^{N} \frac{\mathrm{d}}{\mathrm{d} k} \ln \xi(k)\right|_{k=k_{i}^{+}}\right\rangle=0=\left\langle\prod_{i=1}^{N} S^{\mathrm{fl}}\left(k_{i}\right)\right\rangle .
$$


Eqs. (16) and (8) imply that every correlation function $\left\langle\Delta^{N} \prod_{i=1}^{N} d^{\mathrm{fl}}\left(k_{i}\right)\right\rangle$ can be expressed [15] as a linear combination of the dimensionless $(P, Q)$ level correlation functions defined by

$$
\begin{aligned}
& \Delta^{P+Q}\left\langle\left.\left.\prod_{p=1}^{P} \frac{\mathrm{d}}{\mathrm{d} k} \ln \xi(k)\right|_{k=k^{+}+\kappa_{p}} \prod_{q=1}^{Q} \frac{\mathrm{d}}{\mathrm{d} k} \ln \xi^{*}(k)\right|_{k=k^{+}-\tilde{\kappa}_{q}}\right\rangle \\
& =\Delta^{P+Q}\left\langle\prod_{p=1}^{P} \frac{\mathrm{d}}{\mathrm{d} k} \ln \xi\left(k^{+}+\kappa_{p}\right) \prod_{q=1}^{Q} \frac{\mathrm{d}}{\mathrm{d} k} \ln \xi^{*}\left(k^{+}-\tilde{\kappa}_{q}\right)\right\rangle .
\end{aligned}
$$

Here $P$ and $Q$ are positive integers. Without loss of generality we take $P \geq Q \geq$ 1. In the second line of Eq. (17) we have used the fact that $k+\kappa_{p}$ and $k-\tilde{\kappa}_{q}$ appear only as arguments of an exponential. We are interested in fluctuations on the scale of the mean level spacing. Accordingly we require

$$
\left\langle d_{R}\right\rangle \kappa_{p},\left\langle d_{R}\right\rangle \tilde{\kappa}_{q} \ll B
$$

This is discussed further in Section 5 below. For the $S$-matrix correlator we have correspondingly

$$
\left\langle\prod_{p=1}^{P} S_{\alpha_{p} \beta_{p}}^{\mathrm{fl}}\left(k+\kappa_{p}\right) \prod_{q=1}^{Q}\left(S_{\gamma_{q} \delta_{q}}^{\mathrm{fl}}\left(k-\tilde{\kappa}_{q}\right)\right)^{\dagger}\right\rangle,
$$

again with $P \geq Q \geq 1$ and with the same bounds (18) on $\kappa_{p}$ and $\tilde{\kappa}_{q}$. The dagger stands for the combination of transposition and complex conjugation.

The average over wave vector $k$ is carried out over an averaging interval that is large compared with the minimum difference between any two bond lengths $L_{b}$ (interval length $k_{0}$ with $k_{0} \rightarrow \infty$ ). In calculating the correlation functions, the sequence of limits is, thus, $\lim _{B \rightarrow \infty} \lim _{k_{0} \rightarrow \infty}$. Because of the incommensurability of the bond lengths $L_{b}$ and because of ergodicity, the average over $k$ is then equivalent to $B$ independent averages over the phase angles $\phi_{b}=k L_{b}$, see Refs. 24, 14, 15. We do not present any details because this fact is extensively discussed in Refs. 14, 17]. Eqs. (16) then follow immediately from an expansion of $\xi(k)$ and of $S^{\mathrm{fl}}(k)$ in powers of $\Sigma^{(B)}$. In what follows, angular brackets always denote phase averages.

We demonstrate the need for incommensurate bond lengths $L_{b}$ by a simple example. For $P=1=Q$ the two factors in the correlation functions (17) and (19) can each be expanded in powers of $\Sigma^{(B)}$. The first of these series is proportional to the right-hand side of Eq. (2) and the second to the Hermitean adjoint of that expression. We write the product as a joint power series in $\Sigma^{(B)}$ and $\Sigma^{(B) \dagger}$. Each term contains an exponential. The exponent is a sum of contributions $\pm i k L_{b}$. Averaging the term over $k$ gives a nonvanishing result only if these contributions mutually cancel. If the $L_{b}$ are incommensurate this is possible only if all contributions $i k L_{b}$ appear pairwise with opposite signs. Under that assumption the color-flavor transformation in Section 3 works and effectively 
resums the result of the averaging procedure. Otherwise (i.e., for commensurate $L_{b}$ 's) it becomes extremely difficult to keep track of all the nonvanishing contributions, not to speak of resumming the series. But incommensurability is not a matter of formal convenience only. Commensurate bond lengths may lead to special states ("topological resonances" [26]) which might affect the spectral statistics. Thus, incommensurability seems a neccessary condition for graphs to be chaotic.

\section{Supersymmetry}

In order to work out the phase averages, we express the correlators in Eqs. (17) and (19) as suitable derivatives of a generating function $\mathcal{G}_{\mathrm{G}}$. That function is then expressed [14, 15] as a superintegral. The phase average is worked out with the help of the color-flavor transformation [25].

\subsection{Generating Function}

For closed graphs we use

$$
\begin{aligned}
\Delta \frac{\mathrm{d}}{\mathrm{d} k} \ln \xi(k) & =\operatorname{Tr}\left(\tilde{\mathcal{W}}^{-1}(-i) \Delta \mathcal{L} \sigma_{1}^{d} \Sigma^{(B)}\right) \\
& =\left.\frac{1}{2} \frac{\mathrm{d}}{\mathrm{d} j} \frac{\operatorname{det}\left(\tilde{\mathcal{W}}-i j \Delta \mathcal{L} \sigma_{1}^{d} \Sigma^{(B)}\right)}{\operatorname{det}\left(\tilde{\mathcal{W}}+i j \Delta \mathcal{L} \sigma_{1}^{d} \Sigma^{(B)}\right)}\right|_{j=0} .
\end{aligned}
$$

The differentiation is with respect to the dimensionless source parameter $j$ at $j=0$. For open graphs we define in directed-bond space the matrices

$$
\begin{aligned}
& \left(\mathcal{S}_{p}\right)_{b d, b^{\prime} d^{\prime}}=\tilde{\mathcal{T}}_{b d, \beta_{p}} \mathcal{T}_{\alpha_{p}, b^{\prime} d^{\prime}}, \\
& \left(\tilde{\mathcal{S}}_{q}\right)_{b d, b^{\prime} d^{\prime}}=\tilde{\mathcal{T}}_{b d, \gamma_{q}} \mathcal{T}_{\delta_{q}, b^{\prime} d^{\prime}} .
\end{aligned}
$$

The elements $S_{\alpha_{p} \beta_{p}}^{\mathrm{fl}}$ and the adjoint elements $\left(S_{\gamma_{q} \delta_{q}}^{\mathrm{f}}\right)^{\dagger}$ of the fluctuating part of the $S$ matrix that appear in the correlator (19) can then be written as

$$
\begin{aligned}
S_{\alpha_{p} \beta_{p}}^{\mathrm{fl}} & =\operatorname{Tr}\left(\mathcal{W}^{-1} \mathcal{S}_{p}\right)=\left.\frac{1}{2} \frac{\mathrm{d}}{\mathrm{d} j} \frac{\operatorname{det}\left(\tilde{\mathcal{W}}+j \sigma_{1}^{d} \mathcal{S}_{p}\right)}{\operatorname{det}\left(\tilde{\mathcal{W}}-j \sigma_{1}^{d} \mathcal{S}_{p}\right)}\right|_{j=0}, \\
\left(S_{\gamma_{q} \delta_{q}}^{\mathrm{fl}}\right)^{\dagger} & =\operatorname{Tr}\left(\mathcal{W}^{-1 \dagger} \tilde{\mathcal{S}}_{q}^{\dagger}\right)=\left.\frac{1}{2} \frac{\mathrm{d}}{\operatorname{d} j} \frac{\operatorname{det}\left(\tilde{\mathcal{W}}^{\dagger}+j \tilde{\mathcal{S}}_{q}^{\dagger} \sigma_{1}^{d}\right)}{\operatorname{det}\left(\tilde{\mathcal{W}}^{\dagger}-j \tilde{\mathcal{S}}_{q}^{\dagger} \sigma_{1}^{d}\right)}\right|_{j=0} .
\end{aligned}
$$

We define the source terms

$$
\begin{aligned}
& \mathcal{I}_{p}=-i \Delta \mathcal{L} \sigma_{1}^{d} \Sigma^{(B)}=\tilde{\mathcal{I}}_{q} \text { for closed graphs } \\
& \mathcal{I}_{p}=\sigma_{1}^{d} \mathcal{S}_{p}, \tilde{\mathcal{I}}_{q}=\sigma_{1}^{d} \tilde{\mathcal{S}}_{q} \text { for open graphs }
\end{aligned}
$$

In the ratios of determinants in Eqs. (20) and (22) we multiply numerator and denominator both by $\operatorname{det}(\exp \{i k L\})$ or by the complex conjugate expression. It is convenient to define

$$
z_{p}=\exp \left\{i \kappa_{p} \mathcal{L}\right\}, \tilde{z}_{q}=\exp \left\{i \tilde{\kappa}_{q} \mathcal{L}\right\}
$$


With these steps we define the generating function $\mathcal{G}_{\mathrm{G}}$ for graphs as

$$
\begin{aligned}
\mathcal{G}_{\mathrm{G}}= & \prod_{p=1}^{P} \frac{\operatorname{det}\left(1-\exp \{i k \mathcal{L}\} z_{p}\left[\sigma_{1}^{d} \Sigma^{(B)}-j_{p} \mathcal{I}_{p}\right]\right)}{\operatorname{det}\left(1-\exp \{i k \mathcal{L}\} z_{p}\left[\sigma_{1}^{d} \Sigma^{(B)}+j_{p} \mathcal{I}_{p}\right]\right)} \\
& \times \prod_{q=1}^{Q} \frac{\operatorname{det}\left(1-\left[\Sigma^{(B) \dagger} \sigma_{1}^{d}-\tilde{j}_{p} \tilde{\mathcal{I}}_{q}^{\dagger}\right] \tilde{z}_{q} \exp \{-i k \mathcal{L}\}\right)}{\operatorname{det}\left(1-\left[\Sigma^{(B) \dagger} \sigma_{1}^{d}+\tilde{j}_{q} \tilde{\mathcal{I}}_{q}^{\dagger}\right] \tilde{z}_{q} \exp \{-i k \mathcal{L}\}\right)} .
\end{aligned}
$$

Eqs. (20) and (22) then show that the $(P, Q)$ correlation functions in Eqs. (17) and (19) can both be written as

$$
(P, Q)=\left.\frac{1}{2^{P+Q}} \prod_{p=1}^{P} \prod_{q=1}^{Q} \frac{\mathrm{d}^{2}}{\mathrm{~d} j_{p} \mathrm{~d} \tilde{j}_{q}} \mathcal{G}_{\mathrm{G}}\right|_{\text {all } j=0} .
$$

Expression (25) allows us to treat open and closed graphs completely in parallel.

\subsection{Phase Average of the Generating Function}

With $p=1, \ldots, P$ and $q=1, \ldots, Q$ we define the supervectors $\phi^{(P)}$ with complex commuting elements $s_{p b d}^{(P)}$ and anticommuting elements $\chi_{p b d}^{(P)}$ and $\phi^{(Q)}$ with

commuting complex elements $s_{q b d}^{(Q)}$ and anticommuting elements $\chi_{q b d}^{(Q)}$. These are combined into a single supervector $\Psi=\left(\phi^{(P)}, \phi^{(Q)}\right)^{T}$ of dimension $4 B(P+Q)$. The anticommuting elements obey $\int \chi \mathrm{d} \chi=(2 \pi)^{-1 / 2}=\int \chi^{*} \mathrm{~d} \chi^{*}$. The integration measure in superspace is the flat Berezinian

$$
\begin{aligned}
\mathrm{d}(\tilde{\Psi}, \Psi)= & \prod_{p=1}^{P} \prod_{b d}^{2 B} \mathrm{~d} \Re\left(s_{p b d}^{(P)}\right) \mathrm{d} \Im\left(s_{p b d}^{(P)}\right) \mathrm{d} \chi_{p b d}^{*(P)} \mathrm{d} \chi_{p b d}^{(P)} \\
& \times \prod_{q=1}^{Q} \prod_{b^{\prime} d^{\prime}}^{2 B} \mathrm{~d} \Re\left(s_{q b^{\prime} d^{\prime}}^{(Q)}\right) \mathrm{d} \Im\left(s_{q b^{\prime} d^{\prime}}^{(Q)}\right) \mathrm{d} \chi_{q b^{\prime} d^{\prime}}^{*(Q)} \mathrm{d} \chi_{q b^{\prime} d^{\prime}}^{(Q)}
\end{aligned}
$$

With $\sigma_{3}^{s}$ the third Pauli spin matrix in two-dimensional Boson-Fermion space, we define the $(4 B)$-dimensional diagonal supermatrices $\mathcal{C}_{p}, \tilde{\mathcal{C}}_{q}, \mathcal{B}_{p}$ and $\tilde{\mathcal{B}}_{q}$ by

$$
\begin{aligned}
& \mathcal{C}_{p}=\exp \{i k \mathcal{L}\} z_{p}\left[\sigma_{1}^{d} \Sigma^{(B)}-j_{p} \sigma_{3}^{s} \mathcal{I}_{p}\right]=\exp \{i k \mathcal{L}\} z_{p} \mathcal{B}_{p}, \\
& \tilde{\mathcal{C}}_{q}=\exp \{i k \mathcal{L}\} \tilde{z}_{q}^{*}\left[\sigma_{1}^{d} \Sigma^{(B)}-\tilde{j}_{q} \sigma_{3}^{s} \tilde{\mathcal{I}}_{q}\right]=\exp \{i k \mathcal{L}\} \tilde{z}_{q}^{*} \tilde{\mathcal{B}}_{q} .
\end{aligned}
$$

We define the block-diagonal supermatrix $\mathcal{C}$ of dimension $4 B(P+Q)$ that carries the matrices $\mathcal{C}_{p}\left(\tilde{\mathcal{C}}_{q}^{\dagger}\right)$ in its first $P$ (in its last $Q$ ) blocks, respectively, and analogously for the block-diagonal supermatrix $\mathcal{B}$. The projections of $\mathcal{B}$ onto the retarded (advanced) sector are denoted by $\mathcal{B}_{+}\left(\mathcal{B}_{-}\right.$, respectively). With these definitions the generating function $\mathcal{G}_{\mathrm{G}}$ is written as a superintegral,

$$
\mathcal{G}_{\mathrm{G}}=\int \mathrm{d}(\tilde{\Psi}, \Psi) \exp \{-\tilde{\Psi}(1-\mathcal{C}) \Psi\}
$$


where $\tilde{\Psi}=\left(\Psi^{*}\right)^{T}$. The development leading to Eq. (29) is specific for the case of unitary symmetry. The case of orthogonal symmetry is slightly more complicated and treated in Refs. [14, 15, 16, 17, 19].

We use Eq. (29) to write the phase average of $\mathcal{G}_{\mathrm{G}}$ as

$$
\begin{aligned}
\left\langle\mathcal{G}_{\mathrm{G}}\right\rangle & =\left.\prod_{b=1}^{B} \int \frac{\mathrm{d} \phi_{b}}{2 \pi} \mathcal{G}_{\mathrm{G}}\right|_{k L_{b}=\phi_{b}} \\
& =\int \mathrm{d}(\tilde{\Psi}, \Psi) \exp \{-\tilde{\Psi} \Psi\} \prod_{b=1}^{B} \int \frac{\mathrm{d} \phi_{b}}{2 \pi} \exp \{\tilde{\Psi} \exp \{i \Phi\} z \mathcal{B} \Psi\} .
\end{aligned}
$$

Here $z$ denotes the diagonal supermatrix with elements $\delta_{b b^{\prime}} \delta_{d d^{\prime}} z_{p}$ in the retarded and $\delta_{b b^{\prime}} \delta_{d d^{\prime}} z_{q}$ in the advanced block, jointly referred to as $z_{+}$and $z_{-}$, respectively. The diagonal supermatrix $\Phi$ has elements $\delta_{d d^{\prime}} \phi_{b}$ for all $p$ and $-\delta_{d d^{\prime}} \phi_{b}$ for all $q$. The color-flavor transformation [25] yields

$$
\begin{aligned}
& \prod_{b=1}^{B} \int \frac{\mathrm{d} \phi_{b}}{2 \pi} \exp \{\tilde{\Psi} \exp \{i \Phi\} z \mathcal{B} \Psi\} \\
& =\int \mathrm{d}(\tilde{Z}, Z) \operatorname{SDet}(1-Z \tilde{Z}) \exp \left\{\tilde{\Psi}\left(\begin{array}{cc}
0 & Z \\
\tilde{Z} & 0
\end{array}\right) z \mathcal{B} \Psi\right\} .
\end{aligned}
$$

The matrix $Z(\tilde{Z})$ is rectangular with elements $\delta_{b b^{\prime}} Z_{p b d s ; q b d^{\prime} s^{\prime}}\left(\delta_{b b^{\prime}} \tilde{Z}_{q b d s ; p b d^{\prime} s^{\prime}}\right.$, respectively). The superindex $s=1,2$ denotes the Bosonic and Fermionic degrees of freedom. The Kronecker deltas show that $Z$ and $\tilde{Z}$ are diagonal in bond space. This fact reflects the argument formulated at the end of Section 2.3 (all contributions $i k L_{b}$ appear pairwise with opposite signs). The integration measure $\mathrm{d}(\tilde{Z}, Z)$ is the flat Berezinian. In Boson-Fermion block notation we have

$$
Z=\left(\begin{array}{ll}
Z_{B B} & Z_{B F} \\
Z_{F B} & Z_{F F}
\end{array}\right), \tilde{Z}=\left(\begin{array}{cc}
\tilde{Z}_{B B} & \tilde{Z}_{B F} \\
\tilde{Z}_{F B} & \tilde{Z}_{F F}
\end{array}\right)
$$

with

$$
\tilde{Z}_{B B}=Z_{B B}^{\dagger}, \tilde{Z}_{F F}=-Z_{F F}^{\dagger} .
$$

Moreover, the eigenvalues of the positive definite Hermitean matrix $-\tilde{Z}_{F F} Z_{F F}$ are smaller than or equal to unity.

The Gaussian integrals over the variables in $\Psi$ can now be done. We find

$$
\left\langle\mathcal{G}_{\mathrm{G}}\right\rangle=\int \mathrm{d}(\tilde{Z}, Z) \exp \{-\mathcal{A}(\tilde{Z}, Z)\}
$$

where

$$
\mathcal{A}(\tilde{Z}, Z)=-\mathrm{S} \operatorname{Tr} \ln (1-Z \tilde{Z})+\mathrm{S} \operatorname{Tr} \ln \left(1-z_{+} \mathcal{B}_{+} Z \mathcal{B}_{-}^{\dagger} z_{-} \tilde{Z}\right) .
$$

Here and in what follows the symbol STr without (with) indices denotes the supertrace taken over all matrix indices (only over the matrix indices indicated). The expression (34) for $\left\langle\mathcal{G}_{\mathrm{G}}\right\rangle$ is exact. 


\subsection{Zero Mode}

We show in Section 3.4 that the effective action $\mathcal{A}$ in Eq. (35) contains a massless mode or zero mode. The mode is defined in terms of the eigenvectors $u_{1}$ and $w_{1}$ (see Eqs. (15)) of the leading eigenvalue $\lambda_{1}=1$ of the Perron-Frobenius operator as $Y=\left(Z, u_{1}\right), \tilde{Y}=\left(w_{1}, \tilde{Z}\right)$ or, explicitly, as

$$
\begin{aligned}
& Y_{p b d s, q b^{\prime} d^{\prime} s^{\prime}}=\delta_{b b^{\prime}} \delta_{d d^{\prime}} \frac{1}{\sqrt{2 B}} \sum_{b^{\prime \prime} d^{\prime \prime}} Z_{p b^{\prime \prime} d^{\prime \prime} s, q b^{\prime \prime} d^{\prime \prime} s^{\prime}}, \\
& \tilde{Y}_{q b d s, p b^{\prime} d^{\prime} s^{\prime}}=\delta_{b b^{\prime}} \delta_{d d^{\prime}} \frac{1}{\sqrt{2 B}} \sum_{b^{\prime \prime} d^{\prime \prime}} \tilde{Z}_{q b^{\prime \prime} d^{\prime \prime} s, p b^{\prime \prime} d^{\prime \prime} s^{\prime}} .
\end{aligned}
$$

The supermatrices $(Y, \tilde{Y})$ are multiples of the unit matrix in directed bond space. We show in Section 3.4 that under suitable conditions on the matrix $\Sigma^{(V)}$, the contributions of all other modes to the correlation functions vanish asymptotically $(B \rightarrow \infty)$. Anticipating that result, we express the average generating functions in terms of the zero-mode contribution only. In that approximation, $\left\langle\mathcal{G}_{\mathrm{G}}\right\rangle$ is

$$
\left\langle\mathcal{G}_{\mathrm{G}}^{(0)}\right\rangle=\int \prod_{p q} \mathrm{~d}\left(\tilde{Y}_{q p}, Y_{p q}\right) \exp \left\{-\mathcal{A}^{(0)}\right\} .
$$

Here $\prod_{p q} \mathrm{~d}\left(\tilde{Y}_{q p}, Y_{p q}\right)$ is the flat Berezinian. The zero-mode contribution to the effective action is

$$
\mathcal{A}^{(0)}=-\mathrm{S} \operatorname{Tr} \ln (1-Y \tilde{Y})+\mathrm{S} \operatorname{Tr} \ln \left(1-z_{+} \mathcal{B}_{+} Y \mathcal{B}_{-}^{\dagger} z_{-} \tilde{Y}\right) .
$$

We use Eqs. (24) and expand $\mathcal{A}^{(0)}$ in powers of $\kappa_{p}$ and $\tilde{\kappa}_{q}$, putting all $j_{p}=0=\tilde{j}_{q}$. Because of the inequalities (18) we keep only terms up to first order. We use Eq. (77). That yields the symmetry-breaking term for graphs,

$$
S B_{\mathrm{G}}=\frac{i \pi}{\Delta} \sum_{p} \kappa_{p} \operatorname{Str}_{s}\left(\frac{1}{1-Y \tilde{Y}}\right)_{p p}+\frac{i \pi}{\Delta} \sum_{q} \tilde{\kappa}_{q} \operatorname{Str}_{s}\left(\frac{1}{1-\tilde{Y} Y}\right)_{q q} .
$$

For open graphs, an additional term arises. It is obtained by putting in $\mathcal{A}^{(0)}$ the incremental wave numbers $\kappa_{p}=0=\tilde{\kappa}_{q}$ and all source terms $j_{p}=0=\tilde{j}_{q}$. Since $\tilde{Y}$ commutes with $\sigma_{1}^{d} \Sigma^{(B)}$ we may use the cyclic invariance of the trace to write the term bilinear in $\sigma_{1}^{d} \Sigma^{(B)}$ and $\left(\sigma_{1}^{d} \Sigma^{(B)}\right)^{\dagger}$ in Eq. (38) as $Y \Sigma^{(B) \dagger} \sigma_{1}^{d} \sigma_{1}^{d} \Sigma^{(B)} \tilde{Y}=$ $Y \Sigma^{(B) \dagger} \Sigma^{(B)} \tilde{Y}=Y \Sigma^{(V) \dagger} \Sigma^{(V)} \tilde{Y}$. In the last of these equations we have switched from directed-bond representation to vertex representation. That is permissible because $Y$ equals a multiple of the unit matrix in directed bond space. We take account of the unitarity deficit of the matrix $\Sigma^{(V)}$ by using Eqs. (13) and (14). We expand the action in powers of $Y$ and $\tilde{Y}$, perform the trace over the bond indices in each term of the series, and resum the result. Combining that with the first term on the right-hand side of Eq. (38) we obtain the channel-coupling term

$$
C C_{\mathrm{G}}=-\sum_{\alpha=1}^{\Lambda} \operatorname{STr}_{p s} \ln \left(1+T^{(\alpha)} \frac{Y \tilde{Y}}{1-Y \tilde{Y}}\right) .
$$


The average generating function is

$$
\left\langle G_{\mathrm{G}}^{(0)}\right\rangle=\int \mathrm{d}(\tilde{Y}, Y)(\ldots) \exp \left\{S B_{\mathrm{G}}+C C_{\mathrm{G}}\right\} .
$$

The dots indicate the source terms (i.e., terms that are linear in every $j_{p}$ and in every $\tilde{j}_{q}$ ). Only these contribute to Eq. (26). They are treated in Section 5 , For closed graphs, the channel-coupling term in Eq. (41) is absent.

\subsection{Massive Modes for Graphs}

\subsubsection{Quadratic Approximation to the Effective Action}

We display the zero mode and the massive modes by expanding the effective action (35) up to second order in the variables $\tilde{Z}, Z$, putting $z_{+}=1=z_{-}$, $\kappa_{p}=0=\tilde{\kappa}_{q}$ and dropping the source terms. That gives [15, 17,

$$
\mathcal{A}(Z, \tilde{Z}) \approx \sum_{p, q} \operatorname{STr}_{b d s}\left\{Z_{p q} \tilde{Z}_{q p}-\sigma_{1}^{d} \Sigma^{(B)} Z_{p q} \Sigma^{(B) \dagger} \sigma_{1}^{d} \tilde{Z}_{q p}\right\} .
$$

Eq. (42) applies to both closed and open graphs although the definitions of the matrix $\Sigma^{(B)}$ in the two cases differ. The matrices $\sigma_{1}^{d}$ and $\Sigma^{(B)}$ are the same in all blocks and do not carry block labels $(p, q)$. The action is a sum over independent blocks $(p, q)$. We consider a single such contribution and omit the labels $(p, q)$ for simplicity. In directional space we decompose

$$
Z=\left(\begin{array}{ll}
Z_{++} & Z_{+-} \\
Z_{-+} & Z_{--}
\end{array}\right)=Z_{\text {diag }}+Z_{\text {non }}
$$

into diagonal and non-diagonal contributions, and correspondingly for $\tilde{Z}$. Insertion of this expression into Eq. (42) generates three terms: (i) a term that is bilinear in $Z_{\text {diag }}$ and $\tilde{Z}_{\text {diag }}$; (ii) a term that is bilinear in $Z_{\text {non }}$ and $\tilde{Z}_{\text {non }}$; (iii) a mixed term.

We first show that the mixed term vanishes 15 . Since $Z_{++}, Z_{+-}, Z_{-+}, Z_{--}$ are all diagonal in the bond index $b$, the claim is established if we show that $\left(\sigma_{1}^{d} \Sigma^{(B)}\right)_{b d_{1}, b^{\prime} d_{1}^{\prime}}\left(\Sigma^{(B) \dagger} \sigma_{1}^{d}\right)_{b^{\prime} d_{2}^{\prime}, b d_{2}}$ vanishes for $d_{1}=d_{2}, d_{1}^{\prime} \neq d_{2}^{\prime}$ and for $d_{1}^{\prime}=d_{2}^{\prime}$, $d_{1} \neq d_{2}$. Since $\sigma_{1}^{d}$ is off-diagonal in directional space, that implies $\Sigma_{b d_{1}, b^{\prime} d_{1}^{\prime}}^{(B)}$ $\times \Sigma_{b^{\prime} d_{2}^{\prime}, b d_{2}}^{(B) \dagger}=0$ or $\Sigma_{b d_{1}, b^{\prime} d_{1}^{\prime}}^{(B)} \Sigma_{b d_{2}, b^{\prime} d_{2}^{\prime}}^{(B) \dagger}=0$ for $d_{1}=d_{2}, d_{1}^{\prime} \neq d_{2}^{\prime}$ and for $d_{1}^{\prime}=d_{2}^{\prime}$, $d_{1} \neq d_{2}$. The argument is the same for both cases, and we consider $d_{1}=d_{2}$ and $d_{1}^{\prime} \neq d_{2}^{\prime}$. We recall that the matrix $\Sigma^{(B)}$ carries the elements $\sigma_{\alpha \beta, \alpha \gamma}$ of the matrices $\sigma^{(\alpha)}$. The pair $\left(b d_{1}\right)$ uniquely determines the pair $(\alpha \beta)$. The first factor in $\Sigma_{b d_{1}, b^{\prime} d_{1}^{\prime}}^{(B)} \Sigma_{b d_{2}, b^{\prime} d_{2}^{\prime}}^{(B) *}$ vanishes unless the pair $\left(b^{\prime}, d_{1}^{\prime}\right)$ determines the pair $(\alpha \gamma)$. But then the pair $\left(b^{\prime}, d_{2}^{\prime}\right)$ with $d_{2}^{\prime} \neq d_{1}^{\prime}$ determines the pair $(\gamma \alpha)$ and the element of the second factor with that index vanishes. This establishes our claim. 
Term (ii) is

$$
\begin{aligned}
& \sum_{b b^{\prime}\left(d_{1} \neq d_{2}\right)\left(d_{1}^{\prime} \neq d_{2}^{\prime}\right)}\left(\sigma_{1}^{d} \Sigma^{(B)}\right)_{b d_{1}, b^{\prime} d_{1}^{\prime}} Z_{b^{\prime}, d_{1}^{\prime} d_{2}^{\prime}}\left(\Sigma^{(B) \dagger} \sigma_{1}^{d}\right)_{b^{\prime} d_{2}^{\prime}, b d_{2}} \tilde{Z}_{b, d_{2} d_{1}} \\
= & \sum_{b b^{\prime}\left(d_{1} \neq d_{2}\right)\left(d_{1}^{\prime} \neq d_{2}^{\prime}\right)} \Sigma_{b\left(-d_{1}\right), b^{\prime} d_{1}^{\prime}}^{(B)} Z_{b^{\prime}, d_{1}^{\prime} d_{2}^{\prime}} \Sigma_{b\left(-d_{2}\right), b^{\prime} d_{2}^{\prime}}^{(B) *} \tilde{Z}_{b, d_{2} d_{1}} .
\end{aligned}
$$

For $d_{1} \neq d_{2}$, the pair $\left(b\left(-d_{1}\right)\right)$ (the pair $\left.\left(b\left(-d_{2}\right)\right)\right)$ determines the pair $(\alpha \beta)$ (the pair $(\beta \alpha)$, respectively). The factor $\Sigma_{b\left(-d_{1}\right), b^{\prime} d_{1}^{\prime}}^{(B)}$ vanishes unless the pair $\left(b^{\prime} d_{1}^{\prime}\right)$ determines a pair $(\alpha \gamma)$. Then the pair $\left(b^{\prime} d_{2}^{\prime}\right)$ with $d_{2}^{\prime} \neq d_{1}^{\prime}$ determines the pair $(\gamma \alpha)$. The result vanishes unless $\beta=\gamma$, i.e., unless $\left(b\left(-d_{1}\right)\right)=\left(b^{\prime} d_{1}^{\prime}\right)$ and $\left(b\left(-d_{2}\right)\right)=\left(b^{\prime} d_{2}^{\prime}\right)$. This determines the only nonvanishing contribution. We have $b=b^{\prime}$ and, therefore, $-d_{1}=d_{2}$.

Collecting terms we obtain

$$
\begin{aligned}
& \mathcal{A}(Z, \tilde{Z}) \approx \operatorname{STr}_{s}\left\{\sum_{b_{1} b_{2} d_{1} d_{2}} Z_{b_{1}, d_{1} d_{1}}(1-\mathcal{F})_{b_{1} d_{1}, b_{2} d_{2}} \tilde{Z}_{b_{2}, d_{2} d_{2}}\right. \\
& \left.+\sum_{b\left(d_{1} \neq d_{2}\right)} Z_{b, d_{2} d_{1}}\left(1-\Sigma_{b d_{2}, b d_{2}}^{(B)} \Sigma_{b d_{1}, b d_{1}}^{(B) *}\right) \tilde{Z}_{b, d_{1} d_{2}}\right\}
\end{aligned}
$$

Here $\mathcal{F}$ is the Perron-Frobenius operator defined in Section 2.2. The last term in Eq. (45) was erroneously omitted in Ref. [15]. To identify the zero mode we first consider closed graphs. We use the eigenvector decomposition $\mathcal{F}_{b d, b^{\prime} d^{\prime}}=$ $\sum_{j=1}^{2 B} \lambda_{j}\left|u_{j}\right\rangle\left\langle w_{j}\right|$ and define, in somewhat symbolic notation, for $j=1, \ldots, 2 B$ the supermatrices $z_{j}=\left\langle Z_{\text {diag }} \mid u_{j}\right\rangle$ and $\tilde{z}_{j}=\left\langle w_{j} \mid \tilde{Z}_{\text {diag }}\right\rangle$. (We treat here the matrices $Z_{b d}$ as elements of a linear vector space while actually they are elements of a coset space. That issue has been addressed in Refs. [27, 28]). Returning to the full set of indices, we write Eq. (45) as

$$
\begin{aligned}
& \mathcal{A}(Z, \tilde{Z}) \approx \sum_{p q}\left\{\sum_{j=2}^{2 B}\left(1-\lambda_{j}\right) \operatorname{STr}_{s}\left(z_{p q, j} \tilde{z}_{q p, j}\right)\right. \\
& \left.+\sum_{b\left(d_{1} \neq d_{2}\right)}\left(1-\Sigma_{b d_{2}, b d_{2}}^{(B)} \Sigma_{b d_{1}, b d_{1}}^{(B) *}\right)_{\operatorname{STr}}\left(Z_{p b d_{2}, q b d_{1}} \tilde{Z}_{q b d_{1}, p b d_{2}}\right)\right\} .
\end{aligned}
$$

The term with $j=1$ is conspicuously absent because $\lambda_{1}=1$. That term defines the zero mode or massless mode $(Y, \tilde{Y})$, see Eqs. (36). The remaining modes carry "masses" $\left(1-\lambda_{j}\right)$ and $\left(1-\Sigma_{b d_{2}, b d_{2}}^{(B)} \Sigma_{b d_{1}, b d_{1}}^{(B) *}\right)$. The occurrence of the zero mode or massless mode is a direct consequence of general properties of the Perron-Frobenius operator $\mathcal{F}$. The mode exists for all graphs, and it requires special treatment. The central question is whether $(Y, \tilde{Y})$ is the only such mode, or whether some of the masses of the other modes vanish.

Before addressing that question we turn to open graphs. We claim that Eq. (46) holds also for open graphs. That is obvious for the last term (even 
though the values of $\Sigma_{b d_{2}, b d_{2}}^{(B)} \Sigma_{b d_{1}, b d_{1}}^{(B) *}$ differ). Some more work is required for the first term on the right-hand side of Eq. (46). We define the projector $\Pi_{1}=$ $\left|u_{1}\right\rangle\left\langle w_{1}\right|$ and the orthogonal projector $\Pi_{\perp}=\sum_{j=2}^{2 B}\left|u_{j}\right\rangle\left\langle w_{j}\right|$ onto two eigenvector spaces of $\mathcal{F}$ for closed graphs (even though these are not eigenvector spaces of $\mathcal{F}$ for open graphs). We insert $1=\Pi_{1}+\Pi_{\perp}$ both in front of and right behind the factor $(1-\mathcal{F})$. That gives four terms. The first term $\Pi_{1}(1-\mathcal{F}) \Pi_{1}$ contributes to the channel-coupling term (40) and is not considered further. Upon diagonalization of $\mathcal{F}$ the fourth term $\Pi_{\perp} \mathcal{F} \Pi_{\perp}$ takes the form of the first term on the right-hand side of Eq. (46). Of the remaining two terms we discuss $\Pi_{\perp}(1-\mathcal{F}) \Pi_{1}=-\Pi_{\perp} \mathcal{F} \Pi_{1}$ (the term $\Pi_{1}(1-\mathcal{F}) \Pi_{\perp}$ is treated analogously). With $u_{1}=(1 / \sqrt{2 B})(1,1, \ldots, 1)^{T}$, we have $\mathcal{F}\left|u_{1}\right\rangle_{b d}=(1 / \sqrt{2 B})\left(\sigma_{1}^{d} \Sigma^{(B)} \Sigma^{(B) \dagger} \sigma_{1}^{d}\right)_{b d, b d}$, and $\Pi_{\perp} \mathcal{F} \Pi_{1}$ is seen to be determined by the unitarity deficit of $\Sigma^{(B)}$. We switch from directed-bond representation to vertex representation. We recall that $\Sigma^{(V)}$ is block-diagonal with the matrices $\sigma^{(\alpha)}$ as diagonal entries. From Eqs. (13) we then have $\left(\sigma_{1}^{d} \Sigma^{(B)} \Sigma^{(B) \dagger} \sigma_{1}^{d}\right)_{b d, b d} \leftrightarrow\left(\sigma_{1}^{d} \Sigma^{(B)} \Sigma^{(B) \dagger} \sigma_{1}^{d}\right)_{\alpha \beta, \alpha \beta}^{(V)}=\sum_{\delta}\left|\sigma_{\alpha \delta}^{(\beta)}\right|^{2}=$ $1-\left|\tau_{\alpha}^{(\beta)}\right|^{2}$. The entire term $-\Pi_{\perp} \mathcal{F} \Pi_{1}$ is then $\sum_{j} m_{j} \operatorname{STr}_{s}\left(\tilde{z}_{j} Y\right)$, with the coupling constant $m_{j}$ given by $m_{j}=(1 / \sqrt{2 B}) \sum_{\alpha \beta} w_{j, \alpha \beta}\left|\tau_{\alpha}^{(\beta)}\right|^{2}$. We show that $m_{j} \rightarrow 0$ for $B \rightarrow \infty$ by proving that $\sum_{j}\left|m_{j}^{2}\right| \rightarrow 0$ for $B \rightarrow \infty$. We use Eq. (14) where $T^{(\beta)}$ with $0 \leq T^{(\beta)} \leq 1$ is the transmission coefficient in channel $\beta$. Using completeness in the form $\sum_{j=2}^{\infty} u_{j, \alpha \beta} w_{j, \alpha^{\prime} \beta^{\prime}}=\delta_{\alpha \alpha^{\prime}} \delta_{\beta \beta^{\prime}}-u_{1, \alpha \beta} w_{1, \alpha^{\prime} \beta^{\prime}}$, we calculate the sum of the $\left|m_{j}^{2}\right|$ as

$$
\sum_{j}\left|m_{j}^{2}\right|=\frac{1}{2 B}\left(\sum_{\alpha \beta}\left|\tau_{\alpha}^{(\beta)}\right|^{4}-\frac{1}{2 B}\left(\sum_{\beta} T^{(\beta)}\right)^{2}\right) .
$$

For fixed $\beta$ we have $\sum_{\alpha}\left|\tau_{\alpha}^{(\beta)}\right|^{4} \leq\left(T^{(\beta)}\right)^{2}$. Therefore, the first term on the right-hand side is positive and bounded by $\Lambda /(2 B)$. The last term is bounded in magnitude by $\Lambda^{2} /(2 B)^{2} \ll \Lambda /(2 B)$. Thus, $\sum_{j}\left|m_{j}^{2}\right| \leq \Lambda /(2 B)$. That result is exact and shows that for $\Lambda$ fixed and $B \rightarrow \infty$, all coefficients $m_{j}$ vanish. Therefore, the weight factor (46) for the Gaussian superintegrals over the massive modes holds also for open graphs.

\subsubsection{Loop Expansion}

Generalizing the approach of Refs. [14, 15] we show that the contribution of massive modes to all $(P, Q)$ correlation functions becomes negligible for $B \rightarrow \infty$. We do so by using the quadratic approximation (46) to the effective action, by expanding the remaining terms containing massive modes in power series, and by evaluating the resulting Gaussian superintegrals.

In calculating the Gaussian superintegrals it must be borne in mind that they differ from ordinary Gaussian integrals. The integration extends over both commuting and anticommuting integration variables. We recall that in the Fermion-Fermion sector, the eigenvalues of the matrices $Z^{\dagger} Z$ are bounded. The same condition is obviously met by the Fermion-Fermion sector of the transformed variables $(Y, \tilde{Y})$ and $\left(z_{j}, \tilde{z}_{j}\right)$ with $j \geq 2$. The actual value of the bound 
is immaterial. It can be changed using supersymmetry and a rescaling of the integration variables. All that matters is that in the Fermion-Fermion sectors, the masless mode $(Y, \tilde{Y})$ and the massive modes $\left(z_{j}, \tilde{z}_{j}\right)$ with $j \geq 2$ require a compact parametrization. An infinite range of integration occurs only for the variables in the Boson-Boson blocks.

That last fact defines the conditions under which the Gaussian superintegrals exist that are obtained from the quadratic approximation (46) to the effective action: All masses must be positive. For the series generated in the loop expansion to converge, that statement must be sharpened. Convergence is assured under the following two conditions. (i) The eigenvalues $\lambda_{j}$ with $j \geq 2$ of the Perron-Frobenius operator must lie within or on the surface of a disk that lies entirely within the unit circle in the complex plane. That is the same condition as stated in Section 2.2 for classical mixing of graphs. It must hold for both closed and open graphs. (ii) In the last term of Eq. (46) that same condition must be met by all terms $\left|\Sigma_{b d_{2}, b d_{2}}^{(B)} \Sigma_{b d_{1}, b d_{1}}^{(B) *}\right|$. Since $\Sigma_{b d, b d}^{(B)} \leftrightarrow \Sigma_{\alpha \beta, \alpha \beta}^{(V)}=\sigma_{\beta \beta}^{(\alpha)}$ we have $\Sigma_{b d_{2}, b d_{2}}^{(B)} \Sigma_{b d_{1}, b d_{1}}^{(B) *} \leftrightarrow \sigma_{\beta \beta}^{(\alpha)} \sigma_{\alpha \alpha}^{(\beta)}$. We, thus, require that in the limit $B \rightarrow \infty$ we have $\left|\sigma_{\beta \beta}^{(\alpha)} \sigma_{\alpha \alpha}^{(\beta)}\right| \leq b<1$ for all $\alpha, \beta$. To interpret that condition let us assume that for some pair of vertices $(\alpha, \beta)$ we have $\left|\sigma_{\beta \beta}^{(\alpha)} \sigma_{\alpha \alpha}^{(\beta)}\right|=1$. Unitarity of $\sigma^{(\alpha)}$ then implies $\sigma_{\beta \gamma}^{(\alpha)}=0$ and $\sigma_{\gamma \beta}^{(\alpha)}=0$ for all $\gamma \neq \beta$, and correspondingly for $\sigma^{(\beta)}$. On the bond $(\alpha \beta)$ the Schrödinger waves are completely backscattered by both vertices $\alpha$ and $\beta$. The bond is, thus, completely disconnected from the rest of the graph. It supports an infinite set of bound states. Condition (ii) excludes the existence of sets of such states. These would modify the spectral fluctuation properties of the graph.

The Gaussian superintegrals over the massive modes defined by Eq. (46) yield unity unless the integrand contains further terms that depend on $z_{j}, \tilde{z}_{j}$, $Z_{\text {non }}, \tilde{Z}_{\text {non. }}$. Such terms are generated by expanding the exponential of the difference $\delta \mathcal{A}$ between the effective action in Eq. (35) and the sum $\left[S B_{\mathrm{G}}+C C_{\mathrm{G}}\right.$ plus the right-hand side of Eq. [46)] in a Taylor series. In the series we keep only terms that are of first order in every one of the $j_{p}$ and $\tilde{j}_{q}$ as only these contribute to the $(P, Q)$ correlation functions in Eq. (26).

We first consider closed graphs. Then the source terms in Eq. (23) contain the factor $\Delta \mathcal{L}$. In the summations over directed bonds we replace the factors $L_{b}$ by the average bond length $\bar{L}$. With $\Delta \bar{L}=\pi / B$, each of the source terms becomes inversely proportional to $B$. That is essential for taking the limit $B \rightarrow \infty$. Under omission of the incremental wave numbers $\kappa_{p}, \tilde{\kappa}_{q}$ the part $\delta \mathcal{A}$ of the action difference $\Delta \mathcal{A}$ that contains the source terms is

$$
\delta \mathcal{A}(Z, \tilde{Z})=\operatorname{Str} \ln \left[1-\left(1+i \mathbf{p}_{+}\right) \sigma_{1}^{d} \Sigma^{(B)} Z\left(\sigma_{1}^{d} \Sigma^{(B)}\right)^{\dagger}\left(1-i \mathbf{p}_{-}\right) \tilde{Z}\right] .
$$

Here

$$
\mathbf{p}_{+}=(\pi / B) j_{+} \sigma_{3}^{s}, \mathbf{p}_{-}=(\pi / B) j_{-} \sigma_{3}^{s},
$$

and $j_{ \pm}$are the projections of the source vector $j$ onto the retarded and the advanced sectors. We note that $\mathbf{p}_{ \pm}$and $\sigma_{d}^{1} \Sigma^{(B)}$ commute. The expansion of 
the exponential containing $\delta \mathcal{A}$ generates three types of terms,

$$
\begin{aligned}
& \mathbf{p}_{+}\left(\sigma_{1}^{d} \Sigma^{(B)}\right) Z\left(\sigma_{1}^{d} \Sigma^{(B)}\right)^{\dagger} \tilde{Z} \\
& \left(\sigma_{1}^{d} \Sigma^{(B)}\right) Z \mathbf{p}_{-}\left(\sigma_{1}^{d} \Sigma^{(B)}\right)^{\dagger} \tilde{Z}, \\
& \mathbf{p}_{+}\left(\sigma_{1}^{d} \Sigma^{(B)}\right) Z \mathbf{p}_{-}\left(\sigma_{1}^{d} \Sigma^{(B)}\right)^{\dagger} \tilde{Z} .
\end{aligned}
$$

From these three terms the source terms for all $(P, Q)$ correlation functions are generated.

In the series generated by expanding $\exp \{\Delta \mathcal{A}\}$ we use the transformation that leads from the matrices $Z_{\mathrm{diag}}, \tilde{Z}_{\mathrm{diag}}$ to the matrices $Y, \tilde{Y}, z_{j}, \tilde{z}_{j}$. Each of the terms in the series is then a product of supertraces, each supertrace containing products of the matrices $Y, \tilde{Y}, z_{j}, \tilde{z}_{j}, Z_{\text {non }}, \tilde{Z}_{\text {non }}$ with intermittent factors $\sigma_{1}^{d} \Sigma^{(B)}$, its Hermitean adjoint, source terms, the incremental wave numbers $\kappa_{p}$ and $\tilde{\kappa}_{q}$, the unitary matrix $U$ that diagonalizes $\mathcal{F}$, and its adjoint. The terms that are of order zero in $z_{j}, \tilde{z}_{j}, Z_{\text {non }}, \tilde{Z}_{\text {non }}$ combine to the source terms indicated by $(.$.$) in$ Eq. (14) and are discussed in Section 5 below. In the remaining terms we focus attention on the Gaussian integration over the supermatrices $z_{j}, \tilde{z}_{j}, Z_{\text {non }}, \tilde{Z}_{\text {non }}$. The integrals obviously vanish unless in each term of the series the matrices $Y, \tilde{Y}$, $z_{j}, \tilde{z}_{j}$, and $Z_{\text {non }}, \tilde{Z}_{\text {non }}$ appear in pairs. Moreover, in every such pair the block indices $(p q)$ on $Y$ (or on $z_{i}$ or on $Z_{\text {non }}$ ) must be the same as the block indices $(q p)$ on $\tilde{Y}$ (or on $\tilde{z}_{j}$ or on $\tilde{Z}_{\text {non }}$, respectively). The integrals also vanish unless supersymmetry is broken in both the retarded and the advanced sector of every such pair by a source term containing the matrix $\sigma_{3}^{s}$. When that condition is not met we say that the integrals vanish because of supersymmetry. For simplicity we focus attention on terms containing only the matrices $Y, \tilde{Y}, z_{j}, \tilde{z}_{j}$, this being the slightly more complicated case. Extension of the argument so as to include the matrices $Z_{\text {non }}, \tilde{Z}_{\text {non }}$ is completely straightforward.

We begin with $P=1=Q$. In the integration over massive modes the terms of order one in $\mathbf{p}_{+}$or $\mathbf{p}_{-}$vanish because of supersymmetry. The only nontrivial contribution to the integrand is bilinear in $\mathbf{p}_{+}$and $\mathbf{p}_{-}$. Under omission of numerical factors of order unity the terms of lowest order in $Z, \tilde{Z}$ are

$$
\begin{aligned}
& \operatorname{STr}\left(\mathbf{p}_{+}\left(\sigma_{1}^{d} \Sigma^{(B)}\right) Z\left(\sigma_{1}^{d} \Sigma^{(B)}\right)^{\dagger} \tilde{Z}\right) \operatorname{STr}\left(\left(\sigma_{1}^{d} \Sigma^{(B)}\right) Z \mathbf{p}_{-}\left(\sigma_{1}^{d} \Sigma^{(B)}\right)^{\dagger} \tilde{Z}\right), \\
& \operatorname{STr}\left(\mathbf{p}_{+}\left(\sigma_{1}^{d} \Sigma^{(B)}\right) Z \mathbf{p}_{-}\left(\sigma_{1}^{d} \Sigma^{(B)}\right)^{\dagger} \tilde{Z}\right) \\
& \operatorname{STr}\left(\left[\mathbf{p}_{+}\left(\sigma_{1}^{d} \Sigma^{(B)}\right) Z\left(\sigma_{1}^{d} \Sigma^{(B)}\right)^{\dagger} \tilde{Z}\right]\left[\left(\sigma_{1}^{d} \Sigma^{(B)}\right) Z \mathbf{p}_{-}\left(\sigma_{1}^{d} \Sigma^{(B)}\right)^{\dagger} \tilde{Z}\right]\right) .
\end{aligned}
$$

In the first term we use the transformation leading to Eq. (46), keep at first only terms of order zero in $(Y, \tilde{Y})$, and obtain

$$
\begin{aligned}
& {\left[\sum_{p q} \sum_{j=2}^{2 B} \operatorname{STr}_{s}\left\{\left(\mathbf{p}_{+}\right)_{p} z_{p q, j} \lambda_{j} \tilde{z}_{q p, j}\right\}\right]} \\
& \times\left[\sum_{p^{\prime} q^{\prime}} \sum_{j^{\prime}=2}^{2 B} \operatorname{STr}_{s}\left\{z_{p^{\prime} q^{\prime}, j} \lambda_{j^{\prime}}\left(\mathbf{p}_{-}\right)_{q^{\prime}} \tilde{z}_{q^{\prime} p^{\prime}, j^{\prime}}\right\}\right] .
\end{aligned}
$$


The integral vanishes because of supersymmetry unless $p=p^{\prime}, q=q^{\prime}, j=j^{\prime}$. For fixed $(p, q)$ we turn to the remaining single sum over $j$. Each term in the sum carries a different superintegral. Each such superintegral is well defined because the integrand is free of singularities and the infinite range of the bosonic integration variables is compensated by the Gaussian cutoff. In each of these integrals we rescale the integration variables so as to remove the factor $\left(1-\lambda_{j}\right)$ in the first term on the right-hand side of Eq. (46). The Berezinian of the matrices $z$ and $\tilde{z}$ is flat and not affected by the scaling. Therefore, the scaling generates a factor $1 /\left(1-\lambda_{j}\right)^{2}$ multiplying the integral. We also write the factors $\lambda_{j}$ and $\lambda_{j^{\prime}}$ in Eq. (52) in front of the superintegral. That gives the factor $\lambda_{j}^{2}$. Aside from a relabelling of the integration variables, the remaining superintegrals are identical for each term in the sum and have the same finite value $\mathcal{I}$. The sum takes the form $\left(\mathcal{I} / B^{2}\right) \sum_{j=2}^{2 B} \lambda_{j}^{2} /\left(1-\lambda_{j}\right)^{2}$. That expression vanishes for $B \rightarrow \infty$ if we use $\left|\lambda_{j}\right| \leq a<1$ for all $j \geq 2$ as required in Section 2.2. Avoiding the explicit calculation of the superintegrals throughout, we use that same method in the calculation of all the expressions that follow. For brevity we will say that "aside from numerical factors" the Gaussian superintegrals yield such and such a series.

The second term (51) is

$$
\sum_{p q} \sum_{j=2}^{2 B} \operatorname{Str}_{s}\left\{\left(\mathbf{p}_{+}\right)_{p} z_{p q, j} \lambda_{j}\left(\mathbf{p}_{-}\right)_{q} \tilde{z}_{q p, j}\right\} .
$$

Aside from a numerical factor, the Gaussian superintegrals over this term with fixed values of $(p, q)$ yield $\left(1 / B^{2}\right) \sum_{j=2}^{2 B} \lambda_{j} /\left(1-\lambda_{j}\right) \rightarrow 0$ for $B \rightarrow \infty$. In the third term (51) we again consider only terms of zeroth order in $Y$ and $\tilde{Y}$. Only those contributions survive where $p=p^{\prime}$ and $q=q^{\prime}$ and where, after the transformation to $z_{j}$ and $\tilde{z}_{j}$, all summation indices $j$ are equal. Except for numerical factors, the Gaussian integration yields for fixed $(p, q)$

$$
\begin{aligned}
& \frac{1}{B^{2}} \sum_{j=2}^{2 B} \frac{1}{\left(1-\lambda_{j}\right)^{2}} \sum_{b_{1} b_{2} b_{3} b_{4}} \sum_{d_{1} d_{2} d_{3} d_{4}}\left\{\left(\sigma_{1}^{d} \Sigma^{(B)}\right)_{b_{1} d_{1}, b_{2} d_{2}} w_{b_{2} d_{2}, j}\left(\sigma_{1}^{d} \Sigma^{(B)}\right)_{b_{2} d_{2}, b_{3} d_{3}}^{\dagger}\right. \\
& \left.\times u_{j, b_{3} d_{3}}\left(\sigma_{1}^{d} \Sigma^{(B)}\right)_{b_{3} d_{3}, b_{4} d_{4}} w_{b_{4} d_{4}, j}\left(\sigma_{1}^{d} \Sigma^{(B)}\right)_{b_{4} d_{4}, b_{1} d_{1}}^{\dagger} u_{j, b_{1} d_{1}}\right\} .
\end{aligned}
$$

Here $u$ and $w$ are the eigenvectors of the PF matrix in Section 2.2. Since $\sum_{b^{\prime} d^{\prime}}\left|\left(\sigma_{1}^{d} \Sigma^{(B)}\right)_{b d, b^{\prime} d^{\prime}}\right|^{2}=1$ and $\sum_{b d} u_{j, b d} w_{b d, j}=1$ for all $j$, the multiple sum over $b_{1}, b_{2}, b_{3}, b_{4}$ and $d_{1}, d_{2}, d_{3}, d_{4}$ is bounded in magnitude, with a bound common to all values of $j$, and the expression (54) vanishes for $B \rightarrow \infty$.

In addition to the terms (51), the Taylor expansion of $\exp \{\Delta \mathcal{A}\}$ generates terms of higher order in $Z$ and $\tilde{Z}$ that are also linear in $p_{+}$and $p_{-}$. After transformation to $z_{j}$ and $\tilde{z}_{j}$ these vanish unless the indices $(p, q, j)$ are the same on all $z_{j}$ and $\tilde{z}_{j}$. For a term involving $n$ pairs $z_{j}, \tilde{z}_{j}$ the integration yields the sum $\left(1 / B^{2}\right) \sum_{j=2}^{2 B} \lambda_{j}^{m} /\left(1-\lambda_{j}\right)^{n}$ where $m \leq n$. All these sums tend to zero for $B \rightarrow \infty$ if $\left|\lambda_{j}\right| \leq a<1$ for all $j \geq 2$. As in expression (54) the remaining factors 
are products of ordinary traces over products of factors $\sigma_{1}^{d} \Sigma^{(B)}$, its Hermitean adjoint, the incremental wave numbers $\kappa_{p}$ and $\tilde{\kappa}_{q}$, and the normalized eigenvectors of $\mathcal{F}$. All these traces are bounded from above because $\sigma_{1}^{d} \Sigma^{(B)}$ and $U$ are unitary. Therefore, the contribution of the massive modes to the $P=1=Q$ correlation function that is of zeroth order in $Y$ and $\tilde{Y}$ vanishes for $B \rightarrow \infty$.

In Ref. [15] a weaker condition was used to ensure the vanishing of the contribution due to the massive modes, see also Ref. 32]. The convergence of $\sum_{j=2}^{2 B} \lambda_{j}^{2} /\left(1-\lambda_{j}\right)^{2}$ can be jeopardized only by eigenvalues $\lambda_{j}$ close to unity. With eigenvalues ordered such that $\left|\lambda_{j}\right| \geq\left|\lambda_{j+1}\right|$ for all $j$ with $\lambda_{1}=1$, in Ref. 15] convergence (and, thereby, vanishing of the contribution of massive modes) was assured by requesting that for small $j$ and $B \rightarrow \infty$ we have $\left|\lambda_{j}\right| \propto$ $B^{-\alpha}$ with $0 \leq \alpha<1 / 2$. However, a term containing $n$ pairs $z_{j}, \tilde{z}_{j}$ with arbitrary positive integer $n$ as considered in the previous paragraph (but not considered in Ref. [15]) would impose the stronger bound $\alpha<1 / n$. That condition becomes meaningless for $n \rightarrow \infty$.

Repeating our arguments for mixed terms containing both the matrices $\left(z_{j}, \tilde{z}_{j}\right)$ and the matrices $(Y, \tilde{Y})$ we find that in all three types of terms that occur for $P=1=Q$ such terms do not arise.

Prior to considering the general case we consider a special term that arises for $P=2, Q=1$. With $p_{1} \neq p_{2}$ it is

$$
\begin{aligned}
& \operatorname{STr}_{b d s}\left(\left(\mathbf{p}_{+}\right)_{p_{2}}\left(\sigma_{1}^{d} \Sigma^{(B)}\right) Z_{p_{2} 1}\left(\sigma_{1}^{d} \Sigma^{(B)}\right)^{\dagger} \tilde{Z}_{1 p_{1}}\left(\sigma_{1}^{d} \Sigma^{(B)}\right) Z_{p_{1} 1}\left(\mathbf{p}_{-}\right)_{1}\right. \\
&\left.\times\left(\sigma_{1}^{d} \Sigma^{(B)}\right)^{\dagger} \tilde{Z}_{1 p_{2}}\right) \operatorname{STr} \operatorname{Tr}_{\mathrm{bds}}\left(\left(\mathbf{p}_{+}\right)_{p_{1}}\left(\sigma_{1}^{d} \Sigma^{(B)}\right) Z_{p_{1} 1}\left(\sigma_{1}^{d} \Sigma^{(B)}\right)^{\dagger} \tilde{Z}_{1 p_{1}}\right) .
\end{aligned}
$$

Supersymmetry is broken in the retarded sector in both the $p_{1}$ and the $p_{2}$ blocks by the factors $\left(\mathbf{p}_{+}\right)_{p_{1}}$ and $\left(\mathbf{p}_{+}\right)_{p_{2}}$. Supersymmetry in the advanced sector is jointly broken for both $Z_{p_{1}, 1}$ and for $\tilde{Z}_{1, p_{2}}$ by the single factor $\left(\mathbf{p}_{-}\right)_{1}$. Therefore, integration over the term (55) does not yield zero automatically because of supersymmetry. The example shows that a single symmetry-breaking matrix $\sigma_{3}^{s}$ may be "shared" by two different $Z$ matrices. That insight is important for the general case $P \geq Q \geq 1$.

We use the transformation to $(z, \tilde{z})$, first disregarding $(Y, \tilde{Y})$. Aside from numerical factors, the term (55) yields $\left(1 / B^{3}\right) \sum_{j j^{\prime}}[\ldots]\left(1-\lambda_{j}\right)^{-2}\left(1-\lambda_{j^{\prime}}\right)^{-1}$. The factor $[\ldots]$ contains the sums over $(b d)$ which again are bounded in magnitude, with a bound common to all values of $j, j^{\prime}$. Hence, the term (55) vanishes for $B \rightarrow \infty$. A new situation arises when we consider contributions of higher order in $\left(z_{p_{1} 1, j}, \tilde{z}_{1 p_{1}, j}\right)$ and $\left(z_{p_{2} 1, j^{\prime}}, \tilde{z}_{1 p_{2}, j^{\prime}}\right)$ with $p_{1} \neq p_{2}$ that share with expression (55) the property of containing two factors $\mathbf{p}_{+}$and one factor $\mathbf{p}_{-}$. Such terms arise in the expansion of $\exp \{\Delta \mathcal{A}\}$ and may carry the factors $z_{p_{1} 1, j}, z_{p_{2} 1, j^{\prime}}, \tilde{z}_{1 p_{1}, j}, \tilde{z}_{1 p_{2}, j^{\prime}}$ in intertwined order so that an evaluation of the ensuing Gaussian superintegrals would not be straightforwardly possible. But the scaling of all integration variables in the matrices $\left(z_{p_{1} 1, j}, \tilde{z}_{1 p_{1}, j}\right)$ (in $\left.\left(z_{p_{2} 2, j^{\prime}}, \tilde{z}_{2 p_{2}, j^{\prime}}\right)\right)$ with the factor $\left(1-\lambda_{j}\right)^{1 / 2}$ (with the factor $\left(1-\lambda_{j^{\prime}}\right)^{1 / 2}$, respectively) works in that case, too, removing the 
masses from the Gaussian term in Eq. (46). In the integrand, the scaling produces for each value of $j$ a factor $\left(1-\lambda_{j}\right)^{-l}$. Here $l$ is the total number of pairs $\left(z_{j}, \tilde{z}_{j}\right)$. We then relabel for all values of $j, j^{\prime}$ the scaled integration variables $z_{j} \rightarrow z_{1}, z_{j^{\prime}} \rightarrow z_{2}, \tilde{z}_{j} \rightarrow \tilde{z}_{1}, \tilde{z}_{j^{\prime}} \rightarrow \tilde{z}_{2}$. The resulting integrals over $\left(z_{1}, \tilde{z}_{1}\right)$, $\left(z_{2}, \tilde{z}_{2}\right)$ are common to all terms in the sum over $j, j^{\prime}$, are denoted by $\mathcal{I}$, and can be pulled out of the double summation over $j, j^{\prime}$. (We keep the notation simple and use here and in what follows the same notation for the remaining superintegrals although these actually have a different value in each case.) As a result we obtain $\left(\mathcal{I} / B^{3}\right) \sum_{j j^{\prime}}[\ldots]\left(1-\lambda_{j}\right)^{-l}\left(1-\lambda_{j^{\prime}}\right)^{-l^{\prime}}$. The symbol [...] has the same meaning as before and possesses a bound common to all values of $j, j^{\prime}$. The superintegrals in the factor $\mathcal{I}$ are convergent. As a result, the contribution of the term (55) vanishes in the limit $B \rightarrow \infty$.

In expression (55) the transformation to $(z, \tilde{z})$ may also produce terms that contain the matrices $(Y, \tilde{Y})$, either in the form $\left(Y_{p 1}, \tilde{Y}_{1 p}\right)$ or in the form $\left(Y_{p 2}, \tilde{Y}_{2 p}\right)$. By way of example we consider the case $\left(Y_{p 2}, \tilde{Y}_{2 p}\right)$. Omitting the block indices and using the unitarity of $\sigma_{1}^{d} \Sigma^{(B)}$ we find for the first supertrace in expression (55) the expression

$$
\sum_{j} \sum_{b b^{\prime} d d^{\prime}} \operatorname{STr}_{s}\left(\left(p_{+}\right) Y\left(\sigma_{1}^{d} \Sigma^{(B)}\right)_{b d, b^{\prime} d^{\prime}}^{\dagger} u_{j, b^{\prime} d^{\prime}} \tilde{z}_{j}\left(\sigma_{1}^{d} \Sigma^{(B)}\right)_{b^{\prime} d^{\prime}, b d} w_{b d, j} z_{j}\left(p_{-}\right)_{1} \tilde{Y}\right) .
$$

Combining that with the second supertrace, we arrive at a single sum over $j$ only, and the term vanishes for $B \rightarrow \infty$ more strongly than when the matrices $(Y, \tilde{Y})$ are absent.

We turn to the general case. The expansion of $\exp \{\Delta \mathcal{A}\}$ in powers of $(Z, \tilde{Z})$ generates terms of arbitrarily high orders. We use the transformation to $(z, \tilde{z})$, first disregarding terms that contain $(Y, \tilde{Y})$. Only terms containing pairs of matrices $\left(z_{j}, \tilde{z}_{j}\right)$ with the same index $j$ and belonging to the same pair of block indices $(p, q)$ contribute to the Gaussian superintegrals. Because of supersymmetry, the resulting expressions contribute to the massive modes only if they carry a sufficient number of factors $\left(p_{+}\right)_{p}$ and $\left(p_{-}\right)_{q}$. Naively one might think that for every set of values $(p, q)$ and $j$ that number is two. However, expression (55) shows that two pairs $\left(z_{p q, j}, \tilde{z}_{q p, j}\right)$ and $\left(z_{p^{\prime} q^{\prime}, j^{\prime}}, \tilde{z}_{q^{\prime} p^{\prime}, j^{\prime}}\right)$ with $j \neq j^{\prime}$ and $(p, q) \neq\left(p^{\prime}, q^{\prime}\right)$ may share a factor $\left(p_{+}\right)_{p}$ or a factor $\left(p_{-}\right)_{q}$, as the case may be. Because of such sharing, the term with the smallest inverse power of $B$ in the multiple sum over block indices $(p, q)$ has the form $\left(\mathcal{I} / B^{K+1}\right) \prod_{n=1}^{K} \sum_{j_{n}=2}^{2 B}(1-\lambda)_{j_{n}}^{-l_{n}}[\ldots]$ where the $l_{n}$ are positive integers. The factor $\mathcal{I}$ arises when we scale all integration variables in the manner described above. The factor [...] contains products of sums over products of matrix elements of $S$, of $u$, and of $w$ and possesses a bound common to all values of $j_{n}$ for all $n$. The factor $1 / B^{K+1}$ accounts for the fact that because of supersymmetry, at least one pair $\left(z_{j}, \tilde{z}_{j}\right)$ must carry both factors $\left(p_{+}\right)_{p}$ and $\left(p_{-}\right)_{q}$. Because of the bounds imposed in Section 2.2 on the eigenvalues $\lambda_{j}$ for $j \geq 2$ every sum over one of the $j$ 's multiplied with $1 / B$ converges, and the additional factor $1 / B$ causes the general term to vanish for $B \rightarrow \infty$. 
We turn to the terms in the expansion of $\exp \{\Delta \mathcal{A}\}$ that are at least linear in $(Y, \tilde{Y})$. Every such term is a single supertrace or a product of supertraces. In the first case the matrices $(Y, \tilde{Y})$ appear intertwined with some matrices $\left(z_{j}, \tilde{z}_{j}\right)$, and a straightforward generalization of the argument used for expression (56) shows that the term vanishes more rapidly than when the $(Y, \tilde{Y})$ are replaced by $\left(z_{j}, \tilde{z}_{j}\right)$. In the case of a product of supertraces some supertraces may contain only the matrices $(Y, \tilde{Y})$. We then focus attention on the remaining factors. These may be of order zero in $(Y, \tilde{Y})$. Then the arguments of the previous paragraph apply. Or the remaining factors contain the matrices $(Y, \tilde{Y})$ intertwined with the matrices $\left(z_{j}, \tilde{z}_{j}\right)$. Then the straightforward generalization of the argument used for expression (56) prevails. In all cases every term in the expansion vanishes for $B \rightarrow \infty$.

In conclusion we have shown that for closed graphs, the contribution of the massive modes $\left(z_{j}, \tilde{z}_{j}\right)$ vanishes for all $(P, Q)$ correlation functions if the spectrum of the $\mathrm{PF}$ operator possesses a gap of finite size. The reason is that every source term effectively carries the factor $1 / B$, see Eqs. (49). The same arguments prevail in the case of the massive modes $\left(Z_{b d, b-d}, \tilde{Z}_{b-d, b d}\right)$ (last term in Eq. (46)) provided that $\left|\sigma_{\beta \beta}^{(\alpha)} \sigma_{\alpha \alpha}^{(\beta)}\right| \leq b<1$ for all $(\alpha, \beta)$, and in the case of mixed terms containing both types of massive modes.

To show that the contribution of massive modes vanishes for open graphs, too, we compare the source terms. According to Eqs. (23) and (49) and except for irrelevant factors, the source terms for closed graphs are given by $(1 / B) \Sigma^{(B)}$ and for open graphs by $\mathcal{S}_{p}$ or $\tilde{\mathcal{S}}_{q}$. According to Eqs. (21), each matrix $\mathcal{S}_{p}\left(\tilde{\mathcal{S}}_{q}\right)$ is the dyadic product of a vector $\mathcal{T}_{\alpha_{p}}$ and a vector $\tilde{\mathcal{T}}_{\beta_{p}}$ (of a vector $\mathcal{T}_{\delta_{q}}$ and a vector $\tilde{\mathcal{T}}_{\gamma_{q}}$, respectively). Therefore, only a single eigenvalue $\rho$ of each of the matrices $\mathcal{S}_{p}$ and $\tilde{\mathcal{S}}_{q}$ differs from zero, with $\rho_{p}=\left(T^{\left(\alpha_{p}\right)} T^{\left(\beta_{p}\right)}\right)^{1 / 2}$ and $\rho_{q}=\left(T^{\left(\gamma_{q}\right)} T^{\left(\delta_{q}\right)}\right)^{1 / 2}$ so that $0 \leq \rho \leq 1$ in all cases. In contradistinction the unitarity of $\Sigma^{(B)}$ for closed graphs implies that all eigenvalues of that matrix have absolute value unity. Therefore, traces of matrix products involving $\mathcal{S}_{p}$ or $\tilde{\mathcal{S}}_{q}$ are generically a factor $1 /(2 B)$ smaller than traces of matrix products involving $\Sigma^{(B)}$. That is the factor needed to make the contribution of the massive modes disappear also for open graphs.

\section{Random-Matrix Approach}

We turn to the $(P, Q)$ correlation functions of random-matrix theory. For systems that are not time-reversal invariant we use the Gaussian Unitary Ensemble (GUE) [29]. We define a generalized generating function $\mathcal{G}_{\mathrm{R}}$ for these functions. We generalize the supersymmetry approach of Refs. [30, 18, 31] and average $\mathcal{G}_{\mathrm{R}}$. We then use the Hubbard-Stratonovich transformation and the saddle-point approximation. For closed systems, the CUE [25] would be an alternative to the GUE. We use the GUE because we investigate both closed and open systems, the latter with arbitrarily strong coupling to the channels.

The complex elements $H_{\mu \nu}$ of the $N$-dimensional Hermitean GUE Hamilto- 
nian matrix $H$ are Gaussian-distributed random variables with zero mean values and second moments $\left\langle H_{\mu \nu} H_{\mu^{\prime} \nu^{\prime}}^{*}\right\rangle=\left(\lambda^{2} / N\right) \delta_{\mu \mu^{\prime}} \delta_{\nu \nu^{\prime}}$. The indices $\mu, \nu$ run from 1 to $N$. With $E$ the energy, the $(P, Q)$ level correlation function for the closed system is defined for integer $P \geq Q \geq 1$ as

$$
d^{(P+Q)}\left\langle\prod_{p=1}^{P} \operatorname{Tr}\left(E^{+}+\varepsilon_{p}-H\right)^{-1} \prod_{q=1}^{Q} \operatorname{Tr}\left(E^{-}-\tilde{\varepsilon}_{q}-H\right)^{-1}\right\rangle .
$$

Here $E$ denotes the energy and $d$ the mean level spacing. The plus (minus) sign indicates an infinitesimal positive (negative) imaginary increment. The angular brackets denote the ensemble average. We are interested in fluctuations on the scale of the mean level spacing. Then with $\rho_{\mathrm{R}}=1 / d$ the mean level density, the energy increments $\varepsilon_{p}$ and $\tilde{\varepsilon}_{q}$ obey

$$
\rho_{\mathrm{R}} \varepsilon_{p} \ll \lambda, \rho_{\mathrm{R}} \tilde{\varepsilon}_{q} \ll \lambda .
$$

The open system is obtained [18, 31] by coupling $\Lambda$ channels $a, b, \ldots$ to the states labeled $\mu$ by complex energy-independent channel-coupling matrix elements $W_{a \mu}=W_{\mu a}^{*}$. These obey $\left(W W^{\dagger}\right)_{a b}=N v_{a}^{2} \delta_{a b}$. The unitary scattering matrix is

$$
S_{a b}=\delta_{a b}-2 \pi i\left[W\left(E-H+i \pi W^{\dagger} W\right)^{-1} W^{\dagger}\right]_{a b} .
$$

As done in Refs. [18, 31] we have suppressed the shift matrix. The $(P, Q) S$ matrix correlation function is given by

$$
\left\langle\prod_{p=1}^{P} S_{a_{p} b_{p}}\left(E+\varepsilon_{p}\right) \prod_{q=1}^{Q}\left(S_{c_{q} d_{q}}\left(E-\tilde{\varepsilon}_{q}\right)\right)^{\dagger}\right\rangle
$$

again with the bounds (58) on $\varepsilon_{p}$ and $\tilde{\varepsilon}_{q}$. We note the strong similarity to the developments in Section 2.3 but also the following difference. Expression (17) represents the correlators of the fluctuating parts of the level density only, while the correlators (57) contain the full level densities, including the average parts $1 / d$. Since $\Im \operatorname{Tr}\left(E^{-}-H\right)^{-1}=\pi \delta(E-H)$ and with the normalization chosen in expressions (57) this amounts to the occurence of terms with value $\pi$ in the final expressions for the correlators. Similarly, the correlators (19) are defined in terms of the fluctuating parts of the $S$-matrix elements for graphs while the definitions in Eqs. (63) and (65) below imply that we actually calculate the RMT correlators of $(S-1)$ and not of $S$ as suggested by Eq. (60). That fact yields additional terms $(\langle S\rangle-1)$ in the final expressions for the RMT correlators. These facts must be borne in mind when we later compare the source terms for graphs and for RMT. We calculate the expressions (57) and the correlators of $(S-1)$ for $N \rightarrow \infty$ in the center of the spectrum where $E=0$ and where the mean level spacing $d$ is given by $d=\pi \lambda / N$. We use standard conventions: In graph theory (RMT) the mean level spacing is denoted by $\Delta$ (by $d$ ) and the mean level density by $d$ (by $\rho_{\mathrm{R}}$, respectively). 


\subsection{Generating Function}

To define the generating function we proceed as in Section 3.1. For the closed system we have

$$
d \operatorname{Tr}\left(E^{+}-H\right)^{-1}=\left.\frac{1}{2} \frac{\mathrm{d}}{\mathrm{d} j} \frac{\operatorname{det}\left(E^{+}-H+d j\right)}{\operatorname{det}\left(E^{+}-H-d j\right)}\right|_{j=0} .
$$

For the open system we define the $N$-dimensional source matrices $\mathcal{S}_{p}$ and $\tilde{\mathcal{S}}_{q}$ with elements

$$
\left(\mathcal{S}_{p}\right)_{\mu \nu}=-2 i \pi W_{\mu a_{p}}^{*} W_{b_{p} \nu},\left(\tilde{\mathcal{S}}_{q}\right)_{\mu \nu}=-2 i \pi W_{\mu a_{q}}^{*} W_{b_{q} \nu} .
$$

Then

$$
\begin{aligned}
S_{a_{p} b_{p}}\left(E+\varepsilon_{p}\right)-\delta_{a b} & =\left.\frac{1}{2} \frac{\mathrm{d}}{\mathrm{d} j} \frac{\operatorname{det}\left(E+\varepsilon_{p}-H+i \pi W^{\dagger} W+j \mathcal{S}_{p}\right)}{\operatorname{det}\left(E+\varepsilon_{p}-H+i \pi W^{\dagger} W-j \mathcal{S}_{p}\right.}\right|_{j=0}, \\
\left(S_{c_{q} d_{q}}\left(E-\tilde{\varepsilon}_{q}\right)\right)^{\dagger}-\delta_{a b} & =\left.\frac{1}{2} \frac{\mathrm{d}}{\mathrm{d} j} \frac{\operatorname{det}\left(E-\tilde{\varepsilon}_{q}-H-i \pi W^{\dagger} W+j \tilde{\mathcal{S}}_{q}^{\dagger}\right)}{\operatorname{det}\left(E-\tilde{\varepsilon}_{q}-H-i \pi W^{\dagger} W-j \tilde{\mathcal{S}}_{p}^{\dagger}\right.}\right|_{j=0}
\end{aligned}
$$

We define the source terms

$$
\begin{aligned}
& \mathcal{I}_{p}=d=\tilde{\mathcal{I}}_{q} \text { for the closed system }, \\
& \mathcal{I}_{p}=\mathcal{S}_{p}, \tilde{\mathcal{I}}_{q}=\tilde{\mathcal{S}}_{q} \text { for the open system }
\end{aligned}
$$

and the generating function $G_{\mathrm{R}}$ for the random-matrix approach as

$$
\begin{aligned}
\mathcal{G}_{\mathrm{R}}= & \prod_{p=1}^{P} \frac{\operatorname{det}\left(E^{+}+\varepsilon_{p}-H+i \pi \delta_{\mathrm{open}} W^{\dagger} W+j_{p} \mathcal{I}_{p}\right)}{\operatorname{det}\left(E^{+}+\varepsilon_{p}-H+i \pi \delta_{\mathrm{open}} W^{\dagger} W-j_{p} \mathcal{I}_{p}\right)} \\
& \times \prod_{q=1}^{Q} \frac{\operatorname{det}\left(E^{-}-\tilde{\varepsilon}_{q}-H-i \pi \delta_{\mathrm{open}} W^{\dagger} W+\tilde{j}_{q} \tilde{\mathcal{I}}_{q}\right)}{\operatorname{det}\left(E^{-}-\tilde{\varepsilon}_{q}-H-i \pi \delta_{\mathrm{open}} W^{\dagger} W-\tilde{j}_{q} \tilde{\mathcal{I}}_{q}\right)} .
\end{aligned}
$$

The factor $\delta_{\text {open }}$ equals zero (one) for the closed (the open) system, respectively. With these definitions the $(P, Q)$ correlation functions in Eqs. (57) and (60) can both be written as

$$
(P, Q)=\left.\frac{1}{2^{P+Q}} \prod_{p=1}^{P} \prod_{q=1}^{Q} \frac{\mathrm{d}^{2}}{\mathrm{~d} j_{p} \mathrm{~d} \tilde{j}_{q}} \mathcal{G}_{\mathrm{R}}\right|_{\text {all } j=0} .
$$

\section{$4.2 \quad$ Supersymmetry}

With $p=1, \ldots, P$ and $q=1, \ldots, Q$ we define the supervectors $\phi^{(P)}$ with complex commuting elements $s_{p \mu}^{(P)}$ and anticommuting elements $\chi_{p \mu}^{(P)}$ and $\phi^{(Q)}$ with commuting complex elements $s_{q \mu}^{(Q)}$ and anticommuting elements $\chi_{q \mu}^{(Q)}$. These are 
combined into a single supervector $\Psi=\left(\phi^{(P)}, \phi^{(Q)}\right)^{T}$ of dimension $2 N(P+Q)$. The anticommuting elements obey $\int \chi \mathrm{d} \chi=(2 \pi)^{-1 / 2}=\int \chi^{*} \mathrm{~d} \chi^{*}$. The integration measure in superspace is the flat Berezinian

$$
\begin{aligned}
\mathrm{d}(\tilde{\Psi}, \Psi)= & \prod_{p=1}^{P} \prod_{\mu=1}^{N} \mathrm{~d} \Re\left(s_{p \mu}^{(P)}\right) \mathrm{d} \Im\left(s_{p \mu}^{(P)}\right) \mathrm{d} \chi_{p \mu}^{*(P)} \mathrm{d} \chi_{p \mu}^{(P)} \\
& \times \prod_{q=1}^{Q} \prod_{\nu=1}^{N} \mathrm{~d} \Re\left(s_{q \nu}^{(Q)}\right) \mathrm{d} \Im\left(s_{q \nu}^{(Q)}\right) \mathrm{d} \chi_{q \nu}^{*(Q)} \mathrm{d} \chi_{q \nu}^{(Q)} .
\end{aligned}
$$

The index $s=1,2$ runs over superspace, the index $t=1,2, \ldots, P+Q$ denotes the retarded $(t \leq P)$ and advanced $(t>P)$ blocks. The generating function can be written as

$$
G_{\mathrm{R}}=\int \mathrm{d}(\tilde{\Psi}, \Psi) \exp \left\{\frac{i}{2} \tilde{\Psi} \mathbf{L}^{1 / 2} \mathbf{D} \mathbf{L}^{1 / 2} \Psi\right\}
$$

where $\tilde{\Psi}=\left(\Psi^{*}\right)^{T}$. Here $\mathbf{L}$ is the third Pauli spin matrix in retarded-advanced space. The matrix $\mathbf{D}$ is block diagonal and given by

$$
\mathbf{D}=\mathbf{E}-\mathbf{H}+\varepsilon+i \mathbf{W}+\mathbf{J} .
$$

We define $\varepsilon_{t}=\varepsilon_{p}$ for $t \leq P$ and $\varepsilon_{t}=\tilde{\varepsilon}_{q}$ for $t=P+q$ and correspondingly for $j_{t}$. We write $\mathcal{I}_{t}=\mathcal{I}_{p}$ for $t \leq P$ and $\mathcal{I}_{t}=\mathcal{I}_{q}^{\dagger}$ for $t=P+q$. Then

$$
\begin{aligned}
& \mathbf{E}=\left\{\delta_{t t^{\prime}} \delta_{\mu \mu^{\prime}} \delta_{s s^{\prime}} E\right\}, \mathbf{H}=\left\{\delta_{t t^{\prime}} \delta_{s s^{\prime}} H_{\mu \mu^{\prime}}\right\}, \varepsilon=\left\{\delta_{\mu \mu^{\prime}} \delta_{s s^{\prime}} L_{t t^{\prime}} \varepsilon_{t}\right\} \\
& \mathbf{W}=\left\{\delta_{\mathrm{open}} \delta_{s s^{\prime}} \pi L_{t t^{\prime}}\left(W^{\dagger} W\right)_{\mu \mu^{\prime}}\right\}, \mathbf{J}=\left\{\delta_{t t^{\prime}} \delta_{\mu \mu^{\prime}} \sigma_{3}^{s} j_{t} \mathcal{I}_{t}\right\}
\end{aligned}
$$

We average $\mathcal{G}_{\mathrm{R}}$ over the ensemble by averaging $\exp \left\{-(i / 2)\left(\tilde{\Psi} \mathbf{L}^{1 / 2} \mathbf{H} \mathbf{L}^{1 / 2} \Psi\right)\right\}$. We have

$$
\left\langle\exp \left\{-(i / 2)\left(\tilde{\Psi} \mathbf{L}^{1 / 2} \mathbf{H} \mathbf{L}^{1 / 2} \Psi\right)\right\}\right\rangle=\exp \left\{\frac{1}{8 N} \mathrm{~S} \operatorname{Tr}\left(A^{2}\right)\right\}
$$

where

$$
A_{t s, t^{\prime} s^{\prime}}=i \lambda\left(\mathbf{L}^{1 / 2}\right)_{t t} \sum_{\mu} \Psi_{t \mu s} \Psi_{t^{\prime} \mu s^{\prime}}^{*}\left(\mathbf{L}^{1 / 2}\right)_{t^{\prime} t^{\prime}}
$$

We insert the result of Eqs. (71, 72) into the expression for $\left\langle\mathcal{G}_{\mathrm{R}}\right\rangle$ and remove the terms that are quartic in the integration variables by the Hubbard-Stratonovich transformation. The remaining Gaussian integrals over $\Psi$ and $\tilde{\Psi}$ can be done. All these steps are standard. With $\boldsymbol{\Sigma}=\left\{\delta_{\mu \mu^{\prime}} \sigma_{t s, t^{\prime} s^{\prime}}\right\}$ the result is

$$
\left\langle\mathcal{G}_{\mathrm{R}}\right\rangle=\int \mathrm{d}[\sigma] \exp \left\{-\frac{N}{2} \operatorname{STr}_{t s}\left(\sigma^{2}\right)-\mathrm{S} \operatorname{Tr} \ln (\mathbf{E}-\lambda \mathbf{\Sigma}+\varepsilon+i \mathbf{W}+\mathbf{J})\right\} \text {. }
$$

The matrix $\sigma$ has the same dimension and the same symmetries as the matrix $A$ in Eq. (72). The symbol d $[\sigma]$ denotes the flat Berezinian. Eq. (73) is exact. 


\subsection{Saddle-Point Approximation}

Putting $\varepsilon=0, \mathbf{W}=0, \mathbf{J}=0$ we vary the exponent in $\mathcal{G}_{\mathrm{R}}$ with respect to the elements of $\boldsymbol{\Sigma}$. That yields the saddle-point equation $\sigma(E-\lambda \sigma)=\lambda$. At the center $E=0$ of the GUE spectrum, the solution of that equation is $\sigma_{s p}=-i T_{0}^{-1} \mathbf{L} T_{0}$. Here $T_{0}$ is given by

$$
T_{0}=\left(\begin{array}{cc}
\left(1+t_{12} t_{21}\right)^{1 / 2} & i t_{12} \\
-i t_{21} & \left(1+t_{21} t_{12}\right)^{1 / 2}
\end{array}\right) .
$$

The matrix $t_{12}\left(t_{21}\right)$ has elements $\left(t_{12}\right)_{p s, q s^{\prime}}\left(\left(t_{21}\right)_{q s, p s^{\prime}}\right.$, respectively). The elements of $\left(t_{12}, t_{21}\right)$ span the saddle-point manifold for the $(P, Q)$ correlation function.

We use the saddle-point approximation in Eq. (73) and expand the exponent up to terms of first order in $\varepsilon$, putting $\mathbf{W}=0, \mathbf{J}=0$. That yields the symmetrybreaking term

$$
S B_{\mathrm{R}}=\frac{i \pi}{d} \sum_{p} \varepsilon_{p} \operatorname{STr}_{s}\left(\left(t_{12} t_{21}\right)_{p p}\right)+\frac{i \pi}{d} \sum_{q} \tilde{\varepsilon}_{q} \operatorname{STr}_{s}\left(\left(t_{21} t_{12}\right)_{q q}\right) .
$$

Similarly we put $\varepsilon=0, \mathbf{J}=0$, expand the logarithm in the exponent of Eq. (73), work out the traces over the level indices $\mu$, and resum the resulting series to obtain the channel-coupling term (present only for the open system)

$$
C C_{\mathrm{R}}=-\sum_{c} \mathrm{STr}_{p s} \ln \left(1+T^{(c)} t_{12} t_{21}\right)
$$

With the assumptions introduced above, the average $S$ matrix is diagonal. The transmission coefficient $T^{(c)}$ in channel $c$ is defined as $T^{(c)}=1-\left|\left\langle S_{c c}\right\rangle\right|^{2}$. As a result, the saddle-point approximation to $\left\langle\mathcal{G}_{\mathrm{R}}\right\rangle$ is given by

$$
\left\langle\mathcal{G}_{\mathrm{R}}\right\rangle_{s p}=\int \mathrm{d} \mu(t)(\ldots) \exp \left\{S B_{\mathrm{R}}+C C_{\mathrm{R}}\right\}
$$

Here the dots indicate the source terms. As in Ref. [18, the invariant measure $\mathrm{d} \mu(t)$ is defined by the transformation from the variables that parametrize $\sigma$ to the ones that parametrize $\left(t_{12}, t_{21}\right)$. It turns out that it is not neccessary to work out $\mathrm{d} \mu(t)$ explicitly.

\subsection{Massive Modes}

Massive modes are those degrees of freedom that do not lie in the saddle-point manifold. As for graphs, these are treated in Gaussian approximation. For the orthogonal case and for $P=1=Q$ it is shown in Ref. [18] that the massive modes lie either in the retarded or in the advanced block. The argument carries through also in the unitary case and for $P \geq Q \geq 1$.

For the closed system $(\mathbf{W}=0)$ we follow Ref. [18]. We put $\varepsilon=0$ and $E=0$ for simplicity and write $\sigma=\sigma_{s p}+\delta \sigma$ where $\delta \sigma$ stands for the massive 
modes. We expand the exponent in Eq. (73) in powers of $\delta \sigma$ and keep only terms up to second order, neglecting the source terms. With $\delta \mathcal{P}=T_{0} \delta \sigma_{s p} T_{0}^{-1}$ and $[\delta \mathcal{P}, \mathbf{L}]=0$ that gives in the exponent the term

$$
-N \operatorname{Tr}_{t s}(\delta \mathcal{P})^{2}
$$

In Ref. [18 it is shown that for $N \rightarrow \infty$ and in the vicinity of the saddle point, the Berezinian for $\delta \mathcal{P}$ is flat. The arguments carry through also for the present case. The source terms for the massive modes are given by the expansion of $N \ln \left(1+i \mathbf{L} \delta \mathcal{P}-i \mathbf{L} T_{0} \mathbf{J} T_{0}^{-1} / \lambda\right)$ in powers of $\mathbf{J}$. With $d=\pi \lambda / N$, the term $\mathbf{J} / \lambda$ is inversely proportional to $N$. The substitution $\delta \mathcal{P} \rightarrow \sqrt{N} \delta \mathcal{P}$ then shows that all source terms (and, therefore, the contributions of massive modes) vanish with some inverse power of $N$. That conclusion, demonstrated at the center $E=0$ of the spectrum, can be shown to hold everywhere except near the end points.

We turn to the open system. Expanding the last term in the exponent of Eq. (73) in powers of $\mathbf{W}$ and resumming we find that for $\varepsilon=0$ the term (78) is replaced by

$$
\begin{aligned}
-(N-\Lambda) \operatorname{STr}(\delta \mathcal{P})^{2}+\sum_{a=1}^{\Lambda} \operatorname{STr}_{t s}\left(\frac{i \mathbf{L} x_{a}}{1+i \mathbf{L} x_{a} \sigma_{s p}} \delta \sigma\right) \\
-\sum_{a=1}^{\Lambda} \operatorname{STr}_{t s}\left(\sigma_{s p} \frac{1}{1+i \mathbf{L} x_{a} \sigma_{s p}} \delta \sigma\right)^{2} .
\end{aligned}
$$

Here $x_{a}=\pi N v_{a}^{2} / \lambda$ is of order unity (not $N$ ). The sums extend over the open channels. The same substitutions as used above, i.e., $\delta \sigma \rightarrow \delta \mathcal{P} \rightarrow \sqrt{N} \delta \mathcal{P}$, then show that for $\Lambda$ fixed and $N \rightarrow \infty$, expression (79) reduces to expression (78). The source terms for the massive modes are now given by the expansion of $N \ln \left(1+i \mathbf{L} \delta \mathcal{P}-i\left(\mathbf{L} T_{0} \mathbf{J} T_{0}^{-1} / \lambda\right)-i\left(\mathbf{L} T_{0} \mathbf{W} T_{0}^{-1} / \lambda\right)\right)$ in powers of $\mathbf{J}$. Eqs. (62) show that the matrices $\mathcal{S}_{t}$ are dyadic products of two vectors and, thus, possess only a single nonvanishing eigenvalue. This makes up for the fact that, in contrast to the case of the closed system, they lack a factor $N^{-1}$. An expansion of the logarithmic term in powers of $\mathbf{W}$ embellishes the source terms $\mathbf{J}$ with factors proportional to powers of $x_{a}$ but does not affect the overall dependence on $N$. It follows that the contribution of massive modes vanishes for $N \rightarrow \infty$ also for open graphs.

We have, thus, shown that the contribution of the massive modes to all $(P, Q)$ correlation functions for the GUE vanish with some inverse power of $N$ as $N \rightarrow \infty$. Therefore, these functions are obtained by differentiation of $\left\langle\mathcal{G}_{R}\right\rangle_{s p}$ with respect to the sources.

\section{Equivalence Proof}

We have demonstrated that for $B \rightarrow \infty$ and $N \rightarrow \infty$ the averaged generating functions $\left\langle\mathcal{G}_{\mathrm{G}}\right\rangle$ and $\left\langle\mathcal{G}_{\mathrm{R}}\right\rangle$ become asymptotically equal to $\left\langle\mathcal{G}_{\mathrm{G}}^{(0)}\right\rangle$ and $\left\langle\mathcal{G}_{\mathrm{R}}\right\rangle_{s p}$, 
respectively. The equality of all $(P, Q)$ correlation functions for quantum graphs and RMT is, thus, shown if we can prove that

$$
\left\langle\mathcal{G}_{\mathrm{G}}^{(0)}\right\rangle=\left\langle\mathcal{G}_{\mathrm{R}}\right\rangle_{s p}
$$

We show this by first constructing a one-to-one map of the RMT saddle-point manifold unto the zero-mode manifold of graphs. We use the transformation

$$
\tau=-i t_{12} \frac{1}{\sqrt{1+t_{21} t_{12}}}, \tilde{\tau}=i t_{21} \frac{1}{\sqrt{1+t_{12} t_{21}}} .
$$

With these definitions, Eq. (74) and the relation $\sigma_{s p}=-i T_{0}^{-1} \mathbf{L} T_{0}$ imply in retarded-advanced representation

$$
\sigma_{s p}=-i\left(\begin{array}{cc}
1 & \tau \\
\tilde{\tau} & 1
\end{array}\right)\left(\begin{array}{cc}
1 & 0 \\
0 & -1
\end{array}\right)\left(\begin{array}{cc}
1 & \tau \\
\tilde{\tau} & 1
\end{array}\right)^{-1}
$$

It is shown in Ref. 25 that (except for the factor $-i$ which is properly taken into account in the subsequent RMT calculations) for a parametrization of $\sigma_{s p}$ of the form (82) the integration measure is the flat Berezinian $\prod_{p q} \mathrm{~d}\left(\tilde{\tau}_{q p}, \tau_{p q}\right)$.

The supermatrix $\tau(\tilde{\tau})$ has nonzero elements $\tau_{p s, q s^{\prime}}\left(\tilde{\tau}_{q s, p s^{\prime}}\right)$ only in the retarded-advanced block (in the advanced-retarded block, respectively). In each subblock labelled $(p, q)$ that supermatrix has dimension two. The integration measure for $(\tau, \tilde{\tau})$ is the flat Berezinian. The Boson-Boson (Fermion-Fermion) blocks of the matrices $\tau$ and $\tilde{\tau}$ are related by $\tilde{\tau}_{B B}=\tau_{B B}^{\dagger}$ and by $\tilde{\tau}_{F F}=-\tau_{F F}^{\dagger}$. The eigenvalues of the positive definite Hermitean matrix $-\tilde{\tau}_{F F} \tau_{F F}$ are smaller than or equal to unity. The $B F$ blocks and the $F B$ blocks carry independent anticommuting integration variables. For each pair of indices $(p, q)$ the pair $\left(\tilde{\tau}_{q p}, \tau_{p q}\right)$ of $2 \times 2$ supermatrices possesses the same symmetry properties as the pair $\left(\tilde{\tau}_{11}, \tau_{11}\right)$ the elements of which span the saddle-point manifold for the $(P=1, Q=1)$ correlation functions. All this follows from arguments of symmetry and convergence detailed in Ref. 18 for the orthogonal case. As a consequence of the color-flavor transformation [25] all these properties are shared by the matrices $Y$ and $\tilde{Y}$. Therefore, there exists a one-to-one map of the two sets of matrices $\left(\tau_{p q}, \tilde{\tau}_{q p}\right)$ and $\left(Y_{p q}, \tilde{Y}_{q p}\right)$ onto each other and we can, without loss of generality, equate these matrices,

$$
\tau_{p q}=Y_{p q}, \tilde{\tau}_{q p}=\tilde{Y}_{q p}
$$

Then the RMT saddle-point manifold coincides with the zero-mode manifold for graphs for all values of $P$ and $Q$. The result (83) is not surprising since both sets of matrices parametrize the extension of Efetov's coset space [30] to the general case of $(P, Q)$ correlation functions.

To complete the proof of Eq. (80) we must show that the integrands are identical. To achieve that we identify $\varepsilon_{p} / d$ with $\kappa_{p} / \Delta$ for $p=1, \ldots, P$ and $\tilde{\varepsilon}_{q} / d$ with $\tilde{\kappa}_{q} / \Delta$ for $q=1, \ldots, Q$. This is necessary because the dynamics of graphs is characterized by the wave number $k$ and that of RMT by the energy $E$. Using 
that and Eqs. (81) and (83) in the expression (75) we find that $S B_{\mathrm{R}}$ becomes equal to $S B_{\mathrm{G}}$ as given by Eq. (45). Comparing the constraints formulated in Eqs. (18) and (58) we note that the spectrum of $k$ values is unbounded while the energy spectrum of RMT is bounded by $4 \lambda$. Both constraints can, therefore, be read as saying that the product of the level density and the incremental wave numbers (energies, respectively) be small compared to the length of the spectrum. In that sense, the two sets of constraints are equivalent.

For open graphs we postulate, in addition, that the number of channels be the same for graphs and for RMT and that $\left\langle S_{a a}\right\rangle$ and $\left\langle S_{\alpha \alpha}\right\rangle$ be pairwise equal for all pairs $a, \alpha=1, \ldots, \Lambda$. That implies pairwise equality of the transmission coefficients $T^{(\alpha)}$ and $T^{(a)}$. For the channel coupling term $C C_{R}$ in Eq. (76) that and the above-mentioned substitutions yield the expression (46) for $C C_{\mathrm{G}}$.

To demonstrate the equality of the source terms, we first address the closed system, neglecting the incremental wave numbers and energies. The relevant term in Eq. (73) is $-\mathrm{S} \operatorname{Tr} \ln \left(1+\sigma_{s p} \mathbf{J} / \lambda\right)$. From Eqs. (70) and (64) we have $\mathbf{J} / \lambda=\sigma_{3}^{s} \pi \mathbf{j} / N$, with $\mathbf{j}=\left(j_{+}, j_{-}\right)^{T}$. With $\sigma_{s p}$ given by Eq. (82) and with the definitions $f=(1-\tilde{\tau} \tau)^{-1}, \tilde{f}=(1-\tau \tilde{\tau})^{-1}$ this is written as

$$
\begin{aligned}
1+\sigma_{s p} \mathbf{J} / \lambda= & \left(\begin{array}{cc}
1-i \sigma_{3}^{s} \pi j_{+} / N & 0 \\
0 & 1+i \sigma_{3}^{s} \pi j_{-} / N
\end{array}\right) \\
& +2 i\left(\begin{array}{cc}
-\tau \tilde{\tau} \tilde{f} \sigma_{3}^{s} \pi j_{+} / N & \tau f \sigma_{3}^{s} \pi j_{-} / N \\
-\tilde{\tau} \tilde{f} \sigma_{3}^{s} \pi j_{+} / N & \tilde{\tau} \tau f \sigma_{3}^{s} \pi j_{-} / N
\end{array}\right) .
\end{aligned}
$$

Denoting the first matrix on the right-hand side by $M$ we multiply Eq. (84) on the right by $M^{-1}$. We take account only of terms linear in the source terms. Therefore multiplication of the second matrix with $M^{-1}$ leaves that matrix unchanged. Moreover, we have $\ln \left[\left(1+\sigma_{s p} \mathbf{J} / \lambda\right) M^{-1}\right]=\ln \left(1+\sigma_{s p} \mathbf{J} / \lambda\right)-\ln M$. Since $M$ does not contain any integration variables it does not contribute to the connected part of the correlation functions. Actually, $M$ represents the contributions due to the average level density mentioned below Eq. (60). Indeed, after differentiation with respect to $j_{p}$ and $j_{q}$ that matrix contributes the expected factors $\pi$. For the comparison with the result for graphs (which accounts only for the fluctuating part of the level density) we omit $M$. All this is equivalent to replacing the matrix $M$ in Eq. (84) by the unit matrix. For arbitrary supermatrices $a, b, c, d$ we use the identity

$$
\mathrm{STr}_{t s} \ln \left(\begin{array}{ll}
a & b \\
c & d
\end{array}\right)=\mathrm{STr}_{t s} \ln \left(a-b d^{-1} c\right)+\mathrm{STr}_{t s} \ln d .
$$

With $d=1+2 i \tilde{\tau} \tau f \sigma_{3}^{s} \pi j_{-} / N$ and $\tilde{d}=1+2 i \tau \tilde{f} \sigma_{3}^{s} \tilde{\tau} \pi j_{-} / N$ we have $\ln d=\ln \tilde{d}$. Thus, expression (85) equals $\ln \left(\tilde{d}\left(a-b d^{-1} c\right)\right)$. Using this we obtain

$$
\begin{array}{r}
-\mathrm{S} \operatorname{Tr} \ln \left(1+\sigma_{s p} \mathbf{J} / \lambda\right)=-\mathrm{S} \operatorname{Tr} \ln \left(\left[\left(1+2 i \tau f \sigma_{3}^{s}\left(\pi j_{-} / N\right) \tilde{\tau}\right)\right.\right. \\
\left.\times\left(1-2 i \tau \tilde{\tau} \tilde{f} \sigma_{3}^{s}\left(\pi j_{+} / N\right)\right)\right]-4\left[\left(1+2 i \tau f \sigma_{3}^{s}\left(\pi j_{-} / N\right) \tilde{\tau}\right)\right.
\end{array}
$$




$$
\left.\left.\times \tau f \sigma_{3}^{s}\left(\pi j_{-} / N\right) \frac{1}{1+2 i \tilde{\tau} \tau f \sigma_{3}^{s}\left(\pi j_{-} / N\right)} \tilde{\tau} \tilde{f} \sigma_{3}^{s}\left(\pi j_{+} / N\right)\right]\right) .
$$

We insert in the last line of this expression behind the first factor $j_{-}$the identity, written as $\tilde{\tau}(\tilde{\tau})^{-1}$. Then the first factor in round brackets commutes with $\tau f \sigma_{3}^{s} \tilde{\tau}$. We use

$$
\left(1+2 i \tau f \sigma_{3}^{s}\left(\pi j_{-} / N\right) \tilde{\tau}\right)(\tilde{\tau})^{-1} \frac{1}{1+2 i \tilde{\tau} \tau f \sigma_{3}^{\mathrm{s}}\left(\pi j_{-} / N\right)}=(\tilde{\tau})^{-1}
$$

and obtain

$$
\begin{aligned}
& -\mathrm{S} \operatorname{Tr} \ln \left(1+\sigma_{s p} \mathbf{J} / \lambda\right)=\mathrm{S} \operatorname{Tr} \ln (1-\tau \tilde{\tau}) \\
& -\mathrm{S} \operatorname{Tr} \ln \left(1-\left(1+2 i \pi \sigma_{3}^{s}\left(j_{+} / N\right)\right) \tau\left(1-2 i \pi \sigma_{3}^{s}\left(j_{-} / N\right)\right) \tilde{\tau}\right) .
\end{aligned}
$$

Here and in Eqs. (89) and (90), the first term on the right-hand side is obviously not a source term. With the identification (83) the right-hand side becomes

$$
\mathrm{S} \operatorname{Tr} \ln (1-Y \tilde{Y})-\mathrm{S} \operatorname{Tr} \ln \left(1-\left(1+2 i \pi \sigma_{3}^{s} j_{+} / N\right) Y\left(1-2 i \pi \sigma_{3}^{s} j_{-} / N\right) \tilde{Y}\right) .
$$

For graphs, we use Eqs. (44), $z_{+}=1=z_{-}$, the implicit definitions of the matrices $\mathcal{B}$ in Eqs. (28), and Eqs. (23). We also use that $\left[\sigma_{1}^{d} \Sigma^{(B)}, Y\right]=0$, and we replace $L_{b}$ by the average value $\sum_{b} L_{b} / B$. That gives

$$
\mathrm{S} \operatorname{Tr} \ln (1-Y \tilde{Y})-\mathrm{S} \operatorname{Tr} \ln \left(1-\left(1+2 i \pi \sigma_{3}^{s} j_{+} /(2 B)\right) Y\left(1-2 i \pi \sigma_{3}^{s} j_{-} /(2 B)\right) \tilde{Y}\right) .
$$

The factors $1 / N$ and $1 /(2 B)$ are the inverses of the dimensions $N$ and $2 B$ of the matrices $H$ and $\Sigma^{(B)}$, respectively. These are sent to infinity after the differentiations in Eqs. (26) and (66) are carried out. Without loss of generality we may, therefore, put $N=2 B$. Then the source terms (89) and (90) are identical.

For open systems, the matrices $\mathbf{W}$ and $\sigma_{1}^{d} \Sigma^{(B)}$ are not related in any obvious way. Therefore, the equality of the source terms can be demonstrated only after the terms involving these matrices have been converted into terms involving the average $S$ matrix and/or the transmission coefficients. For the random-matrix approach we use the last term in Eq. (73) taken at the saddle point, putting $\varepsilon=0$ and $E=0$. In the calculations that follow we repeatedly suppress terms of order zero in $\mathbf{J}$ without mention as these are fully taken into account by the channel-channel coupling term (176). In the term

$$
-\mathrm{S} \operatorname{Tr} \ln \left(1+\frac{1}{1+i \lambda^{-1} \sigma_{s p} \mathbf{W}} \lambda^{-1} \sigma_{s p} \mathbf{J}\right)
$$

we expand the denominator in powers of $\mathbf{W}$, use $\left(W W^{\dagger}\right)_{a b}=\delta_{a b} N v_{a}^{2}$, and resum the result. With $x=\left\{\delta_{a b} \pi^{2} v_{a}^{2} / d\right\}$ that gives

$$
-\mathrm{S} \operatorname{Tr} \ln \left(1+\frac{1}{1+i x \sigma_{s p} \mathbf{L}} \lambda^{-1} \sigma_{s p} \mathbf{J}\right) \text {. }
$$


We expand the logarithm in powers of $\mathbf{J}$, use the definitions (70), (64), and (62), perform the trace over the level index $\mu$ and resum the series. We define in channel space (not in level space) the matrix $\mathbf{j}$ with elements $j_{p} \delta_{a a_{p}} \delta_{b b_{p}}$ in the retarded sector and $j_{q} \delta_{a \tilde{b}_{q}} \delta_{b \tilde{a}_{q}}$ in the advanced sector. That gives

$$
-\mathrm{STr}_{a t s} \ln \left(1-\frac{1}{1+i x \sigma_{s p} \mathbf{L}} 2 i x \sigma_{s p} \mathbf{L} \sigma_{3}^{s} \mathbf{j}\right) .
$$

We write this as $-\mathrm{S} \operatorname{Tr} \ln \left(1+i x \sigma_{s p} \mathbf{L}-2 i x \sigma_{s p} \mathbf{L} \sigma_{3}^{s} \mathbf{j}\right)$ with the convention that the channel index on $x$ is determined by the closest factor $\mathbf{j}$ to the right of $x$. We follow the steps that lead from Eq. (84) to Eq. (89). Effectively this amounts in Eq. (88) to the replacements $i \pi \sigma_{3}^{s} j_{+} / N \rightarrow[-x /(1+x)]+2 \sigma_{3}^{s} j_{+} x /(1+x)^{2}$ and $-i \pi \sigma_{3}^{s} j_{-} / N \rightarrow[-x /(1+x)]+2 \sigma_{3}^{s} j_{-} x /(1+x)^{2}$. For fixed $a$ the resulting terms $1-2 x_{a} /\left(1+x_{a}\right)$ are equal to the elements $\left\langle S_{a a}\right\rangle$ of the average $S$ matrix. With the transmission coefficients given by $T^{(a)}=4 x_{a} /\left(1+x_{a}\right)^{2}$ and with $\tau \rightarrow Y$, $\tilde{\tau} \rightarrow \tilde{Y}$ we thus obtain

$$
-\mathrm{STr}_{a t s} \ln \left(1-\left(\langle S\rangle+T \sigma_{3}^{s} \mathbf{j}_{+}\right) Y\left(\langle S\rangle+T \sigma_{3}^{s} \mathbf{j}_{-}\right) \tilde{Y}\right) .
$$

We have defined $\mathbf{j}_{+}=j_{+} \delta_{a a_{p}} \delta_{b b_{p}}$ and correspondingly for $\mathbf{j}_{-}$. The matrices $\langle S\rangle$ and $T$ are diagonal in channel space with elements $\left\langle S_{a a}\right\rangle$ and $T^{(a)}$, respectively. The analogue of the matrix $M$ (first term on the right-hand side of Eq. (84)) is now given by $\left(1+(\langle S\rangle-1) \sigma_{3}^{s} \mathbf{j}\right)$. After differentiation with respect to $j_{p}$ and $j_{q}$, that matrix contributes terms of the form $\langle S\rangle-1$ to the $S$-matrix correlators. Such terms must arise because the $S$-matrix correlators for the RMT case are by construction (see Eq. (63) and the remark below Eq. (60) ) averages over products of elements of $(S-1)$. With $S-1=(\langle S\rangle-1)+S^{\mathrm{fl}}$ they contain factors $(\langle S\rangle-1)$. We disregard these contributions and, thus, the analogue of $M$ because we aim at comparing the source terms for $S^{\mathrm{fl}}$ for RMT and for graphs.

For open graphs we use Eq. (44), the implicit definitions (28) for the matrices $\mathcal{B}$, and the definitions (23). The source terms have the form

$$
-\mathrm{S} \operatorname{Tr} \ln \left(1-\left(\sigma_{1}^{d} \Sigma^{(B)}-\sigma_{3}^{s} j_{+} \mathcal{I}_{+}\right) Y\left(\left(\Sigma^{(B)}\right)^{\dagger} \sigma_{1}^{d}-\sigma_{3}^{s} j_{-} \mathcal{I}_{-}^{\dagger}\right) \tilde{Y}\right) .
$$

Since $\sigma_{1}^{d}$ commutes with $\sigma_{3}^{s} j_{ \pm}$and $Y, \tilde{Y}$ and since $\left(\sigma_{1}^{d}\right)^{2}=1$ this is equal to

$$
-\mathrm{S} \operatorname{Tr} \ln \left(1-\left(\Sigma^{(B)}-\sigma_{3}^{s} j_{+} \mathcal{S}_{+}\right) Y\left(\left(\Sigma^{(B)}\right)^{\dagger}-\sigma_{3}^{s} j_{-} \mathcal{S}_{-}^{\dagger}\right) \tilde{Y}\right) .
$$

We go to the vertex representation, $\Sigma^{(B)} \rightarrow \Sigma^{(V)}$ and the associated changes $S \rightarrow S^{(V)}, \tilde{S} \rightarrow \tilde{S}^{(V)}$. This is permissible because $j_{ \pm}, \sigma_{3}^{s}, Y$, and $\tilde{Y}$ do not depend on directed bond indices. We recall that $\Sigma^{(V)}$ is block diagonal, the matrices $\sigma^{(\alpha)}$ occupying the diagonal blocks. For $\alpha>\Lambda$ these are unitary, and the source terms in these blocks vanish. That leaves us with

$$
-\operatorname{STr}_{\alpha s t} \ln \left(1-\left(\Sigma^{(B)}-\sigma_{3}^{s} j_{+} \mathcal{S}_{+}\right) Y\left(\left(\Sigma^{(B)}\right)^{\dagger}-\sigma_{3}^{s} j_{-} \mathcal{S}_{-}^{\dagger}\right) \tilde{Y}\right) .
$$


The trace extends only over the blocks with $\alpha \leq \Lambda$. In each such block we diagonalize $\sigma \rightarrow U_{1} \sigma_{\operatorname{diag}} U_{2}$, with unitary matrices $U_{1}, U_{2}$. As shown in Ref. [16, all diagonal elements but the first one of $\sigma_{\text {diag }}^{(\alpha)}$ have magnitude unity, the first one being given by $-\rho^{(\alpha)}$ (we suppress here a phase factor which cancels anyway). Each of the source terms $S^{(V)}$ and $\tilde{S}^{(V)}$ is the dyadic product of two vectors. Upon transforming $S^{(V)} \rightarrow U_{1}^{\dagger} S^{(V)} U_{2}^{\dagger}$ and $\tilde{S}^{(V)} \rightarrow U_{2} \tilde{S}^{(V)} U_{1}$, each of these vectors has a single nonvanishing element only. As a result all elements of the transformed matrices $U_{1}^{\dagger} S^{(V)} U_{2}^{\dagger}$ and $U_{2} \tilde{S}^{(V)} U_{1}$ but the $(1,1)$ element vanish. The latter has the value $\left(T^{\left(\alpha_{p}\right)}\right)^{1 / 2}\left(T^{\left(\beta_{p}\right)}\right)^{1 / 2}=T^{1 / 2} \delta_{\alpha \alpha_{p}} \delta_{\beta \beta_{p}} T^{1 / 2}$ in the retarded space and $\left(T^{\left(\delta_{q}\right)}\right)^{1 / 2}\left(T^{\left(\gamma_{q}\right)}\right)^{1 / 2}=T^{1 / 2} \delta_{\alpha \delta_{q}} \delta_{\beta \gamma_{q}} T^{1 / 2}$ in the advanced space. Here $T$ is a diagonal matrix in channel space with elements $T^{(\alpha)}$. With $\mathbf{j}_{+}=j_{+} \delta_{\alpha \alpha_{p}} \delta_{\beta \beta_{p}}$ and $\mathbf{j}_{-}=j_{-} \delta_{\alpha \delta_{q}} \delta_{\beta \gamma_{q}}$ expression (97) becomes

$$
-\operatorname{STr}_{\alpha s t} \ln \left(1-\left(\langle S\rangle+T^{1 / 2} \sigma_{3}^{s} \mathbf{j}_{+} T^{1 / 2}\right) Y\left(\langle S\rangle+T^{1 / 2} \sigma_{3}^{s} \mathbf{j}_{-} T^{1 / 2}\right) \tilde{Y}\right) .
$$

Expanding this into a Taylor series, using in each term that $T$ commutes with $Y$ and $\langle S\rangle$, using the cyclic invariance of the trace, and resumming we obtain

$$
-\operatorname{STr}_{\alpha s t} \ln \left(1-\left(\langle S\rangle+T \sigma_{3}^{s} \mathbf{j}_{+}\right) Y\left(\langle S\rangle+T \sigma_{3}^{s} \mathbf{j}_{-}\right) \tilde{Y}\right),
$$

in perfect agreement with expression (94). This completes the proof of Eq. (80).

\section{Summary and Conclusions}

The combination of the results derived in the present paper with those obtained in Refs. 19, 17] amounts to a complete proof of the BGS conjecture for the cases of orthogonal and unitary symmetry. We have not considered the symplectic case. The proof holds in the limit $B \rightarrow \infty$ of infinite graph size for simple connected graphs with the following three properties. (i) The bond lengths must be incommensurate. (ii) The eigenvalue +1 of the Perron-Frobenius operator must be separated by a finite gap from the rest of the spectrum. (iii) For all $(\alpha, \beta)$ the elements of the vertex scattering matrices must obey $\left|\sigma_{\beta \beta}^{(\alpha)} \sigma_{\alpha \alpha}^{(\beta)}\right| \leq b<$ 1.

The proof given in the present paper is tailored to the unitary case. It involves the following steps. (a) The $(P, Q)$ correlation functions for levels and $S$-matrix elements are witten as derivatives of generating functions. These are expressed as superintegrals. The average over wave number $k$ is performed with the help of property (i) and of the color-flavor transformation. These steps are exact and yield Eqs. (34) and (35) for the averaged generating functions. (b) Expansion of the effective action in Eq. (35) up to terms of second order in the integration variables and transformation to the eigenvector representation of the Perron-Frobenius operator yields the bilinear form (46). In that form the mode corresponding to eigenvalue $\lambda_{1}=1$ is absent. That mode is identified as the zero 
mode $(Y, \tilde{Y})$. (c) The contribution of the remaining modes is calculated using the loop expansion. Under conditions (ii) and (iii) the contributions of the resulting Gaussian superintegrals to every $(P, Q)$ correlation function vanish for $B \rightarrow \infty$. In that limit, the generating functions are entirely determined by the zero mode.

(iv) The equivalence with RMT is established in terms of a one-to-one map of the zero-mode manifold for graphs onto the saddle-point manifold for RMT, and of an identification of all terms in the generating functions. With a slight change of notation and under corresponding conditions on the vertex matrices, our proof carries over to the orthogonal case. Our results hold asymptotically as we have only shown that every contribution of the massive modes vanishes individually in the limit $B \rightarrow \infty$. Therefore, we cannot calculate leading-order corrections in $1 / B$.

In contrast to previous approaches [14, 15, 16, 17, 19] we have not used the saddle-point approximation. Direct use of the zero mode makes the proof stringent, simple, and transparent. We have removed the restriction to completely connected graphs used earlier [16, 17, 19. In view of earlier work on the twopoint function [14, 15, 16, 17, our result is probably expected although it would seem conceivable that differences between graphs and RMT might have existed which, while absent for the two-point functions, would systematically increase with increasing $P$ and $Q$. We have shown that such differences do not exist.

We believe that our derivation and result are of general interest. The PerronFrobenius operator is bistochastic and has a single eigenvalue $\lambda_{1}=1$. We have shown that this fact directly implies the existence of the zero mode. The zeromode manifold represents the generalization to arbitrary values of $P$ and $Q$ of Efetov's coset spaces [30] for unitary or orthogonal symmetry. Aside from the incremental wave numbers and, for open graphs, from the strengths of the couplings to the open channels, the zero-mode part (411) of the generating functions carries no information on the graph actually considered. The zero mode is universal and entirely governed by symmetry. These results are relevant in the limit $B \rightarrow \infty$ where the contributions of the massive modes disappear with inverse powers of $B$, and where the generating functions for graphs and for RMT coincide. That agreement validates the BGS conjecture for graphs.

We discuss our three essential assumptions. (i) Incommensurability of the bond lengths $L_{b}$ guarantees ergodicity and makes it possible to calculate the average over wave number $k$ in terms of an average over independent phases $\phi_{b}=k L_{b}$. That allows us to show that the BGS conjecture holds for every individual graph with incommensurate bond lengths. Alternatively we might consider an ensemble of graphs with a distribution of statistically independent bond lengths $L_{b}$. Averaging over that distribution would likewise yield an average over independent phases $\phi_{b}$. Thus, the BGS conjecture holds on average for an ensemble of graphs irrespective of any condition on bond lengths. Therefore, incommensurability of the bond lengths $L_{b}$ is a less stringent requirement than our conditions (ii) and (iii). For individual graphs with bond lengths that are partly commensurate we do, of course, expect deviations from RMT fluctuation properties. (ii) Classically, the existence of a gap in the spectrum of the Perron-Frobenius operator guarantees that the graph is mixing. In the quan- 
tum context condition (ii) ensures that contributions of the modes $\left(z_{j}, \tilde{z}_{j}\right)$ to the loop expansion vanish asymptotically. While sufficient, condition (ii) may not be necessary for the proof of the BGS conjecture. A weaker condition (powerlaw suppression [32, 15, 33, 34] of the eigenvalue density near $\lambda_{1}=1$ ) might suffice to guarantee classical chaos (albeit not mixing), uniform spreading of eigenfunctions over the graph, and even disappearance of the contributions of the massive modes for $B \rightarrow \infty$ provided the loop expansion could be technically avoided. We have not addressed these questions. (iii) For all $\alpha, \beta$ the diagonal elements of the vertex scattering matrices obey $\left|\sigma_{\beta \beta}^{(\alpha)} \sigma_{\alpha \alpha}^{(\beta)}\right| \leq b<1$. That assumption excludes the formation of bound states on single bonds that are completely separated from the rest of the graph. Such states might affect the spectral fluctuation properties.

The conditions (ii) and (iii) are physically plausible, and deviations from universal behavior are expected if one is violated. Take condition (ii), for instance, and let us consider two completely connected simple chaotic graphs $g_{1}$ and $g_{2}$ that are connected with each other by a single bond. In the limit of infinite graph size we expect that the single bond plays an ever diminishing role, and that the spectral properties are dominated by the zero-mode manifolds for $g_{1}$ and $g_{2}$. We conjecture that the spectrum is a superposition of two GOE (or two GUE) spectra with weak repulsion between levels from $g_{1}$ and those from $g_{2}$. Another case is a chain of completely connected simple chaotic graphs with few bonds connecting only neighboring members in the chain. Here Anderson localization might be expected, causing strong deviations from Wigner-Dyson statistics.

Are there graphs that satisfy conditions (ii) and (iii) and if so, how numerous are they? As for the first part of the question, there are examples in the orthogonal case where the spectrum of the Perron-Frobenius operator possesses a gap [35. If time-reversal invariance is violated by a magnetic field that affects the phases of the bond propagators but not the matrices $\sigma^{(\alpha)}$, the spectrum of the Perron-Frobenius operator is unaffected and the gap persists in the unitary case. Concerning condition (iii), bound states on single bonds with total reflection at the adjacent vertices require very special boundary conditions. Therefore, we conjecture that condition (iii) is satisfied for most Hermitean boundary conditions imposed at the vertices. The second part of the question requires a deeper understanding of the relationship between the boundary conditions at the vertices and the spectrum of the Perron-Frobenius operator [36, 13, 37, 32]. We consider this an important challenge for future investigations.

The symmetry-breaking terms for graphs (for RMT) are obtained by expanding the effective action in powers of the wave-number increments $\kappa_{p}, \tilde{\kappa}_{q}$ (the energy increments $\varepsilon_{p}, \tilde{\varepsilon}_{q}$, respectively). With the identifications defined in Section 5 both terms agree to lowest order in these increments. Inspection shows that the terms of next order do not agree. Therefore, the agreement of the fluctuation properties of graphs with those of RMT is limited to a wavenumber interval defined by the range of validity of the approximations used. With $\kappa$ representing any of the $\kappa_{p}$ or $\tilde{\kappa}_{q}$, we have $\operatorname{expanded} \exp \left\{i \kappa L_{b}\right\}$ in a Tay- 
lor series and kept terms of order zero and one only. The neglect of higher-order terms is justified if $\kappa L_{b} \ll 1$ for all $b$ or, equivalently, if $\kappa \ll 1 / L_{\text {max }}$. With $\bar{L}$ the average bond length, the dimensionless variable $\kappa / \Delta$ must, therefore, obey $\kappa / \Delta \ll B \bar{L} / L_{\max }$. That bound tends to infinity with $B$, and so does the range of agreement of the $(P, Q)$ correlation functions for graphs and for RMT. This situation differs from the case of the semiclassical approximation where the spectral fluctation properties agree with RMT predictions in an energy interval defined by the shortest periodic orbit.

In Refs. 38, 39, 40, use of the classical PF operator was advocated as a sufficient means to characterize the spectral fluctuation properties of chaotic quantum systems. For the modes $\left(z_{j}, \tilde{z}_{j}\right)$ (Eq. (46)) the present work confirms that suggestion: Their masses are determined by the eigenvalues of the PF operator. However, knowledge of the PF operator is not sufficient for the nondiagonal massive modes. Their masses depend on the matrix elements of the vertex scattering matrix $\Sigma^{(B)}$, a quantum operator, see Eq. (46).

Acknowledgements. The authors are grateful to E. Bogomolny, P. Cejnar, S. Gnutzmann, P. LeBoeuf, P. Kurasov, J. Kvasil, and U. Smilansky for useful comments. Thanks are due to an anonymous referee for helpful and constructive criticism. ZP acknowledges support by the Czech Science Foundation under Project No P203 - 13 - 07117S.

\section{References}

[1] O. Bohigas, M. J. Giannoni, and C. Schmit, Phys. Rev. Lett. 52, 1 (1984).

[2] S. W. McDonald and A. N. Kaufman, Phys. Rev. Lett. 42, 1189 (1979).

[3] G. Casati, F. Valz-Gris, and I. Guarneri, Lett. Nuovo Cimento Soc. Ital. Fis. 28, 279 (1980).

[4] M. V. Berry, Ann. Phys. (N.Y.) 131, 163 (1981).

[5] F. J. Dyson, J. Math. Phys. 3, 1199 (1962).

[6] F. Haake, Quantum Signatures of Chaos, 3rd Edition, Springer-Verlag, Heidelberg/New York (2010).

[7] M. Sieber, K. Richter, Physica Scripta T 90, 128 (2001).

[8] S. Müller, S. Heusler, P. Braun, F. Haake, and A. Altland, Phys. Rev. Lett. 93, 014103 (2004).

[9] S. Müller, S. Heusler, P. Braun, F. Haake, and A. Altland, Phys. Rev. E 72, 046207 (2005).

[10] S. Heusler, S. Müller, A. Altland, P. Braun, and F. Haake, Phys. Rev. Lett. 98, 044103 (2007). 
[11] S. Müller, S. Heusler, A. Altland, P. Braun, and F. Haake, New J. Phys. 11, 103025 (2009).

[12] M. Gutzwiller, Chaos in Classical and Quantum Mechanics, Springer, New York 1990.

[13] T. Kottos and U. Smilansky, Ann. Phys. (N.Y.) 274, 76 (1999).

[14] S. Gnutzmann and A. Altland, Phys. Rev. Lett. 93, 194101 (2004).

[15] S. Gnutzmann and A. Altland, Phys. Rev. E 72, 056215 (2005).

[16] Z. Pluhař and H. A. Weidenmüller, Phys. Rev. Lett. 110, 034101 (2013).

[17] Z. Pluhař and H. A. Weidenmüller, Phys. Rev. E 88, 022902 (2013).

[18] J. J. M. Verbaarschot, H. A. Weidenmüller, and M. R. Zirnbauer, Phys. Rep. 129, 367 (1985).

[19] Z. Pluhař and H. A. Weidenmüller, Phys. Rev. Lett. 112, 144102 (2014).

[20] S. Gnutzmann and U. Smilansky, Adv. Phys. 55, 527 (2006).

[21] T. Kottos and U. Smilansky, J. Phys. A: Math. Gen. 36, 3501 (2003).

[22] F. Barra and P. Gaspard, Phys. Rev. E 63, 066215 (2001).

[23] P. Pakonski, K. Zyczkowski, and M. Kus, J. Phys. A 34, 9303 (2001).

[24] F. Barra and P. Gaspard, J. Stat. Phys. 101, 283 (2000).

[25] M. R. Zirnbauer, J. Phys. A: Math. Gen. 29, 7113 (1996).

[26] S. Gnutzmann, H. Schanz, and U. Smilansky, Phys. Rev. Lett. 110, 094101 (2013).

[27] M. R. Zirnbauer, in: I. V. Lerner, J. P. Keating, and D. E. Khmelnitzkii, editors, Supersymmetry and Trace Formulae: Chaos and Disorder, Proceedings of a NATO ASI held in Cambridge, UK, 1997, Plenum Publishers, 1999, page 153 .

[28] A. Altland, S. Gnutzmann, F. Haake, and T. Micklitz, arXiv:1412.5336.

[29] M. L. Mehta, Random Matrices, 3rd edition, Academic Press, , New York (2004).

[30] K. B. Efetov, Adv. Phys. 32, 53 (1983).

[31] Y. V. Fyodorov, D. V. Savin, and H.-J. Sommers, J. Phys. A 38, 10731 (2005).

[32] G. Tanner, J. Phys. A 34, 8485 (2001). 
[33] S. Gnutzmann, J. P. Keating, and F. Piotet, Phys. Rev. Lett. 101, 264102 (2008).

[34] S. Gnutzmann, J. P. Keating, and F. Piotet, Ann. Phys. (N.Y.) 325, 2595 (2010).

[35] J. M. Harrison, U. Smilansky, and B. Winn, J. Phys. A: Math. Theor. 40, 14181 (2007).

[36] U. Smilansky, arXiv:chao-dyn.9611002

[37] G. Berkolaiko, J. Phys. A: Math. Gen. 34, L319 (2001).

[38] O. Agam, B. L. Altshuler, and A. V. Andreev, Phys. Rev. Lett. 75, 4389 (1995).

[39] A. V. Andreev, O. Agam, B. D. Simons, and B. L. Altshuler, Phys. Rev. Lett. 76, 3947 (1996).

[40] A. V. Andreev, B. D. Simons, O. Agam, and B. L. Altshuler, Nucl. Phys. B 482, 536 (1996). 